\title{
Passivity-based control of a class of Blondel-Park transformable electric machines*
}

\author{
PER J. NICKLASSONं, ROMEO ORTEGAII and \\ GERARDO ESPINOSA-PÉREZ§
}

Keywords: Passivity-based control, electric machines, physical systems

\begin{abstract}
In this paper we study the viability of extending, to the general rotating electric machine's model, the passivity-based controller method that we have developed for induction motors. In this approach the passivity (energy dissipation) properties of the motor are taken advantage of at two different levels. First, we prove that the motor model can be decomposed as the feedback interconnection of two passive subsystems, which can essentially be identified with the electrical and mechanical dynamics. Then, we design a torque tracking controller that preserves passivity for the electrical subsystem, and leave the mechanical part as a "passive disturbance". In position or speed control applications this procedure naturally leads to the well known cascaded controller structure which is typically analyzed invoking time-scale separation assumptions. A key feature of the new cascaded control paradigm is that the latter arguments are obviated in the stability analysis. Our objective in this paper is to characterize a class of machines for which such a passivity-based controller solves the output feedback torque tracking problem. Roughly speaking, the class consists of machines whose nonactuated dynamics are well damped and whose electrical and mechanical dynamics can be suitably decoupled via a coordinate transformation. The first condition translates into the requirement of approximate knowledge of the rotor resistances to avoid the need of injecting high gain into the loop. The latter condition is known in the electric machines literature as Blondel-Park transformability, and in practical terms it requires that the air-gap magnetomotive force must be suitably approximated by the first harmonic in its Fourier expansion. These conditions, stemming from the construction of the machine, have a clear physical interpretation in terms of the couplings between its electrical, magnetic and mechanical dynamics, and are satisfied by a large number of practical machines. The passivity-based controller presented here reduces to the well known indirect vector controller for current-fed induction machines. Our developments constitute an extension, to voltage-fed machines, of this de facto standard in industrial applications. Furthermore, our analysis provides it with a solid theoretical foundation.
\end{abstract}

\section{Introduction}

One of the main driving forces of control theory has traditionally been the characterization of classes of systems for which a certain control objective is achievable. This allows us to identify the system structural constraints-usually expressed in terms

*This work was supported in part by the Norwegian Research Council under contract 100651/410.

$\uparrow$ Author to whom all correspondence should be addressed.

\$SINTEF Electronics and Cybernetics Automatic Control, N-7034 Trondheim, Norway, Per.J.Nicklasson@ecy.sintef.no

qLaboratoire des Signaux et Systèmes CNRS-ESE, Plateau de Moulton, 91192 Gif-surYvette, France, rortega@Iss.supelec.fr

§Universidad de Mexico, Instituto de Ingeniería, P.O. Box 70-472, D.F., Mexico, gerardoe@servidor.ùnam.mx

(C1997 IEEE. Reprinted, with permission from IEEE Transactions on Automatic Control, Vol. 42, pp. 629-647, 1997. 
of system invariants-which are compatible with the desired performance. A fairly complete theory along this line is now available both for linear [7], [36] and nonlinear systems [24], [52]. From the practical viewpoint the interest in identifying the systems invariants is to be able to attach to them some physical interpretation that can be used in the controller design. Although in the linear case this characterization is readily available and practically useful, for instance in the form of effective transmission delays or sensor actuator couplings, their nonlinear counterparts developed to date seem to be less suitable for practical applications. A good example of this state of affairs concerns the fundamental problem of stabilizability. Stabilizable systems have been identified via geometric conditions for linearization [24], [52], [45] or transformation to particular (triangular, pure feedback) forms [49], [30], [46]. (See [26], [59], [30], [46] for recent surveys and other characterizations of stabilizable systems). In the authors' opinion there are several shortcomings to these results. First, the geometric conditions seem to be hard to verify in practical applications and are usually unrelated with the physical constraints of the system. Second, the control laws typically require full state measurement, hence the inclusion of observers for their practical implementation. Finally, since they are derived neglecting the systems physical constraints, they are often not well defined in all the operating regimes.

An alternative approach to systems stabilization that, to a certain extent, overcomes the aforementioned shortcomings is to relate stabilizability of the system to the possibility of rendering it passive via feedback. This idea, first advanced in an adaptive control context in [60], has been explored in ${ }^{1}$, e.g., [54], [9], [62], [27]. The relevance of using passivity as a building block for control systems design stems, not just from the important role this concept plays in systems stability analysis and its close connection with the systems physics, but also in the invariance of this property vis a vis feedback interconnection [13]. This aspect is particularly relevant in our work where the key steps in the controller design are the decomposition of the system into passive subsystems, and the choice of a feedback control that preserves the passivity of the closed loop.

Our motivation in this paper is to contribute, if modestly, to the development of a stabilization theory for physical systems that incorporates at a fundamental level the systems physical structure. To achieve this objective we restrict ourselves to a particular, but practically very important, class of systems-the general rotating electric machine [47], [71], and establish conditions for stabilizability which are interpretable in terms of the systems physics. Our main contribution is the definition of a class of machines such that the output feedback torque tracking problem can be solved via a passivity-based controller. Roughly speaking, the class consists of machines whose nonactuated (rotor) dynamics are suitably damped and whose electrical and mechanical dynamics can be "partially" decoupled, via a coordinate transformation. Machines satisfying the latter condition are known in the electric machines literature as Blondel-Park transformable [41]. In practical terms it requires that the air-gap magnetomotive force be suitably approximated by the first harmonic in a Fourier expansion. These two conditions, stemming from the construction of the machine, have a clear physical interpretation in terms of materials and the couplings

\footnotetext{
${ }^{1}$ Some efforts to reinterpret from a passivity perspective the currently popular "backstepping technique" [32], [31] derived for systems with special forms are reported in [43], [35], [30]. See also [59] for some interesting connections between input to state stability and dissipativity.
} 
between its electrical, magnetic and mechanical dynamics, and are satisfied by a large number of practical machines.

The idea of passivity-based controller designs is to reshape the systems natural energy and inject the required damping in such a way that the control objective is achieved. Expected advantages of this approach are the enhanced robustness properties and lack of (controller calculation) singularities, properties which stem from the fact that cancellation of systems nonlinearities is avoided. The technique has its roots in classical mechanics [21], [2] and was introduced in control theory in the seminal paper [66], see also [58] for an early reference. This method has been instrumental in the solution of several robotics [55], [3], [37], [1], induction motor [16], [56] and power electronics [63] problems which were untractable with other stabilization techniques. See also the recent interesting book [68].

The passivity-based controller design we present here is the extension of a class of Blondel-Park transformable machines of the one used in [16] for induction motors, and proceeds as follows. First, we carry out a decomposition of the system dynamics as a feedback interconnection of passive subsystems where the outputs of the forward subsystem are the measurable and the regulated outputs. A lemma that gives conditions on the energy function under which this is possible is given. Second, we design an inner feedback loop that, via the injection of a nonlinear damping term, ensures the controlled subsystem defines a strictly passive map from control signals to measurable outputs. Third, the passivity-based technique is applied to this subsystem leaving the feedback subsystem as a "passive perturbation". As explained in [16] this last step involves the definition of the desired closed-loop energy function whose associated (target) dynamics evolves on a subspace of the state space insuring zero error tracking ${ }^{2}$. The overall procedure leads to a nonlinear dynamic output feedback controller that ensures global asymptotic torque tracking with internal stability.

In position or speed control applications an outer loop is added to the torque controller [56]. As pointed out in [39], there appears to be universal agreement that this structure, (with a fast inner current loop that can be regarded as creating impressed currents to the stator windings necessary to achieve the desired torque specified by the outer loops), is the most effective control scheme for electrical drives. This procedure naturally leads to the well known cascaded controller structure which is typically analyzed invoking time-scale separation assumptions. A key feature of the new cascaded control paradigm is that stability is now established without these arguments. See [57] for further discussions on this topic.

The remainder of the paper is organized as follows. In Section 2 we present a simple, but important, passive systems feedback decomposition lemma. The class of systems considered in the paper - the generalized electric machine's model-is given in Section 3 , and in Section 4 we formulate the control problem and describe the design procedure. Conditions for strict passifiability of the electrical subsystem are established in Section 5. In Section 6 and 7, we explain how the passivity based approach is used to achieve current and torque tracking, and in Section 8 we present the main results in this paper. Examples and experimental results are given in Section 9 and Section 10. Finally, concluding remarks are given in Section 11.

\footnotetext{
${ }^{2}$ It is worth pointing out that to overcome the singularity problems on a backstepping-based controller for induction motors the authors in [12] borrowed this last step from [56].
} 


\section{Notation}

See also [13]. $\mathscr{R}$-field of real numbers; $\|\cdot\|$-Euclidean norm; $(\cdot)^{T}$-transposition; $\mathscr{I}_{n}-n \times n$ identity matrix; $\mathrm{e}^{(\cdot)}$-matrix exponential;

$$
\frac{d}{d t}(\cdot)=(\cdot) \text {-total time derivative; }
$$

$\mathscr{L}_{2}^{n}, \mathscr{L}_{\infty}^{n}, \mathscr{L}_{2 e}^{n}, \mathscr{L}_{\infty e}^{n}$-spaces of $n$-dimensional square integrable, essentially bounded functions and their extensions; a causal system $\Sigma: \mathscr{L}_{2 e}^{n} \mapsto \mathscr{L}_{2 e}^{n}$ is said to be passive (resp. output strictly passive) if $\exists \alpha \geq 0$ (resp. $>0$ ) and $\exists \beta \in \mathscr{R}$ such that

$$
\int_{0}^{\gamma} u^{T}(t)(\Sigma u)(t) d t \geq \alpha \int_{0}^{\gamma}\|\Sigma u\|^{2} d t+\beta, \forall u \in \mathscr{L}_{2 e}^{n}, \forall \gamma \geq 0 .
$$

\section{Passive subsystems feedback decomposition}

We consider nonlinear electromechanical systems described by the Euler-Lagrange equations of motion [47]

$$
\frac{d}{d t}\left[\frac{\partial \mathscr{L}(q, \dot{q})}{\partial \dot{q}}\right]-\frac{\partial \mathscr{L}(q, \dot{q})}{\partial q}=Q
$$

where ${ }^{3} \mathscr{L}=\mathscr{J}^{*}(\dot{q}, q)-\mathscr{V}(q)$ is the system Lagrangian, $\mathscr{J}^{*}: \mathscr{R}^{2 n} \rightarrow \mathscr{R}$ is the total system coenergy-state function, $\mathscr{V}: \mathscr{R}^{n} \rightarrow \mathscr{R}$ is the total system energy-state function, $q \in \mathscr{R}^{n}$ are the generalized coordinates and $Q \in \mathscr{R}^{n}$ are the external (dissipative and control) forces. We assume that the system energy-state function (potential energy) is bounded from below.

From the well known energy balance equation of Euler-Lagrange systems [68]

$$
\underbrace{H(t)-H(0)}_{\text {stored energy }}=\underbrace{\int_{0}^{t} \dot{q}^{T} Q d s}_{\text {dissipated }+ \text { supplied }}
$$

where

$$
H:=\left(\frac{\partial \mathscr{L}}{\partial \dot{q}}\right)^{T} \dot{q}-\mathscr{L}
$$

is the overall system's total energy, it follows immediately that the system (2.1) defines a passive operator $\Sigma: \mathscr{L}_{2 e}^{n} \rightarrow \mathscr{L}_{2 e}^{n}: Q \mapsto \dot{q}$.

\section{Lemma 2.1.}

Assume the Lagrangian of (2.1) can be decomposed in the form

$$
\mathscr{L}=\mathscr{L}_{e}\left(q_{e}, \dot{q}_{e}, q_{m}\right)+\mathscr{L}_{m}\left(q_{m}, \dot{q}_{m}\right)
$$

\footnotetext{
${ }^{3}$ To simplify the notation, here and throughout the rest of the paper we will omit the arguments of the functions, giving them explicitly only when they are first defined.
} 
where $q:=\left[q_{e}^{T}, q_{m}^{T}\right]^{T}$ with $q_{e} \in \mathscr{R}^{n_{e}}$ and $q_{m} \in \mathscr{R}^{n_{m}}$. Then, $\Sigma$ can be represented as the negative feedback interconnection of two passive subsystems

$$
\begin{gathered}
\Sigma_{e}: \mathscr{L}_{2 e}^{n} \rightarrow \mathscr{L}_{2 e}^{n}:\left[\begin{array}{c}
Q_{e} \\
-\dot{q}_{m}
\end{array}\right] \mapsto\left[\begin{array}{c}
\dot{q}_{e} \\
y
\end{array}\right] \\
\Sigma_{m}: \mathscr{L}_{2 e}^{n_{m}} \rightarrow \mathscr{L}_{2 e}^{n_{m}}:\left(y+Q_{m}\right) \mapsto \dot{q}_{m}
\end{gathered}
$$

where

$$
y:=\frac{\partial \mathscr{L}_{e}}{\partial q_{m}}
$$

is the subsystems coupling signal, and $Q:=\left[Q_{e}^{T}, Q_{m}^{T}\right]^{T}$ with $Q_{e} \in \mathscr{R}^{n_{e}}, Q_{m} \in \mathscr{R}^{n_{m}}$.

\section{Proof}

Using the Euler-Lagrange procedure to derive the equations of motion, we get

$$
\begin{gathered}
\frac{d}{d t}\left[\frac{\partial \mathscr{L}_{e}}{\partial \dot{q}_{e}}\right]-\frac{\partial \mathscr{L}_{e}}{\partial q_{e}}=Q_{e} \\
\frac{d}{d t}\left[\frac{\partial \mathscr{L}_{m}}{\partial \dot{q}_{m}}\right]-\frac{\partial \mathscr{L}_{m}}{\partial q_{m}}=Q_{m}+y
\end{gathered}
$$

Evaluating the total time derivative of $\mathscr{L}_{e}$ we get

$$
\dot{\mathscr{L}}_{e}=\frac{\partial \mathscr{L}_{e}^{T}}{\partial q_{e}} \dot{q}_{e}+\frac{\partial \mathscr{L}_{e}^{T}}{\partial \dot{q}_{e}} \ddot{q}_{e}+\frac{\partial \mathscr{L}_{e}^{T}}{\partial q_{m}} \dot{q}_{m}
$$

Noting that

$$
\frac{\partial \mathscr{L}_{e}^{T}}{\partial \dot{q}_{e}} \ddot{q}_{e}=\frac{d}{d t}\left[\frac{\partial \mathscr{L}_{e}^{T}}{\partial \dot{q}_{e}} \dot{q}_{e}\right]-\frac{d}{d t}\left[\frac{\partial \mathscr{L}_{e}^{T}}{\partial \dot{q}_{e}}\right] \dot{q}_{e}
$$

inserting this into (2.4), using (2.2) and rearranging the terms, we obtain

$$
\frac{d}{d t} H_{e}=Q_{e}^{T} \dot{q}_{e}-\dot{q}_{m}^{T} y
$$

where

$$
H_{e}\left(q_{e}, \dot{q}_{e}, q_{m}\right):=\frac{\partial \mathscr{L}_{e}^{T}}{\partial \dot{q}_{e}} \dot{q}_{e}-\mathscr{L}_{e}
$$

Using the arguments of Section 9.1 in [11] we can show that $H_{e}$ is the total energy of the subsystem $\Sigma_{e}$. Integrating from 0 to $\gamma$ and setting $\beta_{e}:=-H_{e}(0)$ proves the passivity of $\Sigma_{e}$.

A similar procedure can be used to establish the passivity of $\Sigma_{m}$, using the energy function

and (2.3).

$$
H_{m}=\frac{\partial \mathscr{L}_{m}^{T}}{\partial \dot{q}_{m}} \dot{q}_{m}-\mathscr{L}_{m}
$$

\section{General Rotating Machine}

\subsection{Model}

In this paper we treat the general rotating machine considered in [41], (see also [74], 
[72]). It consists of a total of $n_{e}$ windings on stator and rotor and we assume ideal symmetrical phases and sinusoidally distributed phase windings. The permeability of the fully laminated cores is assumed to be infinite and saturation, iron losses, end winding and slot effects are neglected. Linear magnetic materials are considered, and we assume that all parameters are constant and known ${ }^{4}$.

Under the assumptions above, application of Gauss' law and Ampere's law leads to the following affine relationship between the flux linkage vector $\lambda=\left[\lambda_{1}, \ldots, \lambda_{n_{e}}\right]^{T}$ and the current vector $\dot{q}_{e}=\left[\dot{q}_{1}, \ldots, \dot{q}_{n_{e}}\right]^{T}$

$$
\lambda=D_{e}\left(q_{m}\right) \dot{q}_{e}+\mu\left(q_{m}\right)
$$

where $q_{m} \in \mathscr{R}$ is the mechanical position of the motor, $D_{e}=D_{e}^{T}>0$ is the $n_{e} \times n_{e}$ multiport inductance matrix of the windings and the vector $\mu$ represents the flux linkages due to the possible existence of permanent magnets. Both $D_{e}$ and $\mu$ are bounded and periodic in $q_{m}$.

If we define as generalized coordinates of the system the total amounts of moving electric charge that has passed any given point on the different phase windings, $q_{i}$, $i=1, \ldots, n_{e}$, and the angular position of the rotor $q_{m}$, we can compute the magnetic-field coenergy (with ' denoting the variable of integration) as [47]

$$
\mathscr{K}_{e}=\sum_{i=1}^{n_{e}} \int_{0}^{\dot{q}_{i}} \lambda_{i}\left(\dot{q}_{i}^{\prime}\right)=\frac{1}{2} \dot{q}_{e}^{T} D_{e} \dot{q}_{e}+\mu^{T} \dot{q}_{e}
$$

and the mechanical kinetic coenergy as

$$
\mathscr{K}_{m}=\frac{1}{2} D_{m} \dot{q}_{m}^{2},
$$

where $D_{m}>0$ is the rotational inertia of the rotor.

Neglecting the capacitive effects in the windings of the motor and considering a rigid shaft, we have that the potential energy $\mathscr{V}$ of the system is only due to the interactions between the magnetic materials in stator and rotor $^{5}$, i.e. $\mathscr{V}=\mathscr{V}\left(q_{m}\right)$. We get the Lagrangian

$$
\mathscr{L}\left(\dot{q}_{e}, \dot{q}_{m}, q_{m}\right)=\frac{1}{2} \dot{q}_{e}^{T} D_{e} \dot{q}_{e}+\mu^{T} \dot{q}_{e}+\frac{1}{2} D_{m} \dot{q}_{m}^{2}-\mathscr{V}\left(q_{m}\right)
$$

To model the external forces we will assume that the dissipative effects are linear time invariant and only due to the resistances in the windings $r_{i} \geq 0, i=1, \ldots, n_{e}$, and the mechanical friction $R_{m} \geq 0$. The control forces are the voltages applied to the windings $u \in \mathscr{R}^{n_{s}}, n_{s} \leq n_{e}$. We consider here fully actuated as well as underactuated machines, that is, machines where the voltages can be applied only to stator windings (e.g., induction motor), or to both stator and rotor windings (e.g., synchronous motor with field windings). Hence, we find convenient to partition the vector of generalized electrical coordinates as $q_{e}=\left[q_{s}^{T}, q_{r}^{T}\right]^{T} \in \mathscr{R}^{n_{e}}, q_{s} \in \mathscr{R}^{n_{s}}, q_{r} \in \mathscr{R}^{n_{r}}, n_{r}=n_{e}-n_{s}$, where the subscripts $s, r$ are used to denote variables related to windings with and without actuation respectively. In the case of underactuated machines the partition coincides with stator and rotor variables as well. Notice however, that there are also machines, like the PM synchronous, PM stepper and variable reluctance motors, where $q_{e}$

\footnotetext{
${ }^{4}$ See [14] for relaxations of the latter restriction.

${ }^{5}$ This energy contribution is zero if there are magnetic materials in only one part (stator or rotor), and the reluctance properties of the other part is uniform.
} 
consists only of stator variables which are directly actuated by the stator voltages, see the examples in Section 3.3. Finally, we assume ${ }^{6}$ that the load torque $y_{L}$ in the mechanical subsystem is of the form

$$
y_{L}\left(q_{m}, \dot{q}_{m}\right)=\left[k_{1}+k_{3} \dot{q}_{m}^{2}\right] \tanh \left(\frac{\dot{q}_{m}}{\rho}\right)+k_{2} \dot{q}_{m}
$$

with a scaling parameter $\rho>0$.

With the considerations above, and applying the Euler-Lagrange equations to (3.2), we obtain the equations of motion of the generalized machine as

$$
\begin{gathered}
D_{e} \ddot{q}_{e}+W_{1}\left(q_{m}\right) \dot{q}_{m} \dot{q}_{e}+W_{2}\left(q_{m}\right) \dot{q}_{m}+R_{e} \dot{q}_{e}=M_{e} u \\
D_{m} \ddot{q}_{m}-y\left(\dot{q}_{e}, q_{m}\right)+R_{m} \dot{q}_{m}=-y_{L}
\end{gathered}
$$

where

$$
W_{1}:=\frac{d D_{e}\left(q_{m}\right)}{d q_{m}}, W_{2}:=\frac{d \mu\left(q_{m}\right)}{d q_{m}}, R_{e}:=\operatorname{diag}\left\{r_{s} \mathscr{I}_{n_{s}}, r_{r} \mathscr{I}_{n_{r}}\right\}, M_{e}:=\left[\begin{array}{c}
\mathscr{I}_{n_{s}} \\
0
\end{array}\right]
$$

with $y$ the generated torque

$$
y=h\left(\dot{q}_{e}, q_{m}\right):=\frac{1}{2} \dot{q}_{e}^{T} W_{1} \dot{q}_{e}+W_{2}^{T} \dot{q}_{e}+\eta\left(q_{m}\right)
$$

and

$$
\eta\left(q_{m}\right)=-\frac{d V}{d q_{m}}
$$

which is also bounded and periodic in $q_{m}$.

Notice that the machine is fully characterized by its dynamic parameters $D:=\operatorname{diag}\left\{D_{e}, D_{m}\right\}, M_{e}, \mu, \eta$ and the dissipation parameters $R:=\operatorname{diag}\left\{R_{e}, R_{m}\right\}$. Therefore, in the sequel we will refer to the system described by (3.4), (3.5), (3.6) as $\Sigma\left(D, R, M_{e}, \mu, \eta\right)$. Similarly, the electrical subsystem (3.4), (3.6) will be denoted by $\Sigma_{e}\left(D_{e}, R_{e}, M_{e}, \mu, \eta\right)$, and the mechanical subsystem (3.5) by $\Sigma_{m}\left(D_{m}, R_{m}\right)$.

\subsection{Remarks}

1. We have derived here the model of the electrical machine using the Lagrangian formalism. It is clear that the same model can be obtained from the application of the basic force laws. Besides its esthetic appeal and generality, variational modeling is the natural framework for passivity-based designs, where energy functions and workless forces must be identified. Furtheremore, as pointed out in [71], we tend to believe that "the long-term value of variational techniques precludes their dismissal merely on the ground of conceptual difficulties or lack of physical insight when employed by the novice".

2. If we introduce the flux ${ }^{7} \lambda$ as defined by (3.1) into (3.4), we get the voltage

${ }^{6}$ The presence of a load torque $y_{L}$ of this form ensures that to every bounded $y$ there exists a bounded $\dot{q}_{m}$. Except from this, as will be shown below, the load torque $y_{L}$ plays no role in the torque tracking problem. Also, as shown in [16] it can be treated as an external disturbance for the speed tracking problem.

${ }^{7}$ We recall that fluxes and currents are the generalized electrical momenta and velocities of the Hamiltonian formalism [71]. 
balance equation $\dot{\lambda}+R_{e} \dot{q}_{e}=M_{e} u$. Particularly useful for further developments is the following relationship between motor fluxes and rotor currents for motors where the rotor windings are short circuited (induction motors)

$$
\dot{\lambda}_{r}+R_{r} \dot{q}_{r}=0
$$

where $\lambda:=\left[\begin{array}{ll}\lambda_{s}^{T}, & \lambda_{r}^{T}\end{array}\right]^{T}$ and $R_{e}=\operatorname{diag}\left\{R_{s}, \quad R_{r}\right\} \quad$ with $R_{s}=r_{s} \mathscr{I}_{n_{s}} \in \mathscr{R}^{n_{s} \times n_{s}}$, $R_{r}=r_{r} \mathscr{I}_{n_{r}} \in \mathscr{R}^{n_{r} \times n_{r}}$. The importance of (3.8) is that it defines a dynamic relationship between $\dot{q}_{r}$ and $\lambda_{r}$ which is unaffected by the control action, hence we will have to take this into account when defining a "desired behaviour" for the machine.

3. An interesting property of $\Sigma\left(D, R, M_{e}, \mu, \eta\right)$ and other magnetic field devices in which currents are of main interest, is that the electric charges are ignorable [71], (also known as cyclic in mechanics [2]). That is, the Lagrangian of the system does not contain $q_{e}$ (although it contains the corresponding currents $\dot{q}_{e}$ ). It must be pointed out that when choosing the form (2.1) of the Euler-Lagrange equations, it is crucial that the currents are expressed in their natural frames (where electric charges can be obtained by integration of currents) to avoid introduction of quasi coordinates. See also [71].

4. For machines with mechanical commutation, the relation between port and rotor currents introduces non-holonomic constraints [71], [50] and the dynamic equations cannot be obtained directly from (2.1) with the given Lagrangian. Instead quasi coordinates could be introduced, and the dynamic equations derived by using the Boltzmann-Hamel [71] or Gaponov [50] form of the Euler-Lagrange equations. These procedures are however quite involved, and the dynamic equation for this class of machines is therefore usually not derived from variational principles, but by the use of basic laws as Faraday's law, Ohm's law and Euler's law. Also, since the Euler-Lagrange procedure is based on energy properties, the dynamic equations for machines with nonlinear magnetics can also be derived by this procedure. The switched reluctance machine is an example of a machine in which the magnetic nonlinearities must be taken into account. We will not consider machines with mechanical commutation or nonlinear magnetics in this paper, but refer the reader to [20] where the latter issue is addressed.

\subsection{Examples}

In [41] several examples of electric machines described by $\Sigma(D, R, \mu, \eta)$ are given. We present here two examples of fully actuated machines, i.e., where $n_{s}=n_{e}$ and $M_{e}=\mathscr{I}_{n_{e}}$.

(i) For the $3 \phi P M$ synchronous motor [33] we have $n_{e}=3$ and the parameters

$$
\begin{gathered}
D_{e}\left(q_{m}\right)= \\
{\left[\begin{array}{ccc}
L_{l s}+L_{A}-L_{B} \cos 2 n_{p} q_{m} & -\frac{1}{2} L_{A}-L_{B} \cos 2\left(n_{p} q_{m}-\frac{\pi}{3}\right) & -\frac{1}{2} L_{A}-L_{B} \cos 2\left(n_{p} q_{m}+\frac{\pi}{3}\right) \\
-\frac{1}{2} L_{A}-L_{B} \cos 2\left(n_{p} q_{m}-\frac{\pi}{3}\right) & L_{l s}+L_{A}-L_{B} \cos 2\left(n_{p} q_{m}-\frac{2 \pi}{3}\right) & -\frac{1}{2} L_{A}-L_{B} \cos 2\left(n_{p} q_{m}+\pi\right) \\
-\frac{1}{2} L_{A}-L_{B} \cos 2\left(n_{p} q_{m}+\frac{\pi}{3}\right) & -\frac{1}{2} L_{A}-L_{B} \cos 2\left(n_{p} q_{m}+\pi\right) & L_{l s}+L_{A}-L_{B} \cos 2\left(n_{p} q_{m}+\frac{2 \pi}{3}\right)
\end{array}\right]}
\end{gathered}
$$




$$
\mu\left(q_{m}\right)=\lambda_{m}\left[\begin{array}{c}
\sin n_{p} q_{m} \\
\sin \left(n_{p} q_{m}-\frac{2 \pi}{3}\right) \\
\sin \left(n_{p} q_{m}+\frac{2 \pi}{3}\right)
\end{array}\right]
$$

where $L_{l s}, L_{A}, L_{B}$ are inductance parameters, $n_{p}$ is the number of pole pairs and $\lambda_{m}$ is the amplitude of the flux linkage established by the permanent magnet.

(ii) The 2 $\phi$ PM stepper motor has $n_{e}=2$ and the following parameters [75]

$$
\begin{gathered}
D_{e}=\left[\begin{array}{ll}
L & 0 \\
0 & L
\end{array}\right] \\
\mu\left(q_{m}\right)=\frac{K_{m}}{N_{r}}\left[\begin{array}{l}
\cos \left(N_{r} q_{m}\right) \\
\sin \left(N_{r} q_{m}\right)
\end{array}\right]
\end{gathered}
$$

$L$ is the self-inductance of each winding, and $K_{m}$ is the torque constant. $N_{r}$ is the number of rotor teeth of same polarity. In this case the torque has a term due to the interaction between the permanent magnet and the magnetic material in the stator (detent torque), and $\eta\left(q_{m}\right)=-K_{D} \sin \left(4 N_{r} q_{m}\right), K_{D} \approx 5-10 \%$ of $K_{m} i_{0}$, where $i_{0}$ is the rated current.

\section{Problem formulation and Design Procedure}

\subsection{Problem formulation}

We will assume here that the currents of the actuated windings $\dot{q}_{s}$ and rotor position $q_{m}$ and velocity $\dot{q}_{m}$ are available for measurement. Also, we will take as basic regulated variable the generated torque $y$, which is however unmeasurable since it depends on the variables $\dot{q}_{r}$. Notice that the motor speed is related to the latter via a simple linear passive operator (3.5). Thus by regulating $y-y_{L}$ and ensuring passivity in closed loop, as can be done in passivity-based control, we can expect to have a good behaviour in the mechanical subsystem with a simple (PI) speed outer loop.

Our control problem can therefore be formulated as follows:

Output feedback torque tracking problem. Consider the $2 n_{e}+2$ dimensional machine model $\Sigma(D, R, M, \mu, \eta)$ with state vector $\left[q_{e}^{T}, \dot{q}_{e}^{T}, q_{m}, \dot{q}_{m}\right]^{T}$, inputs $u \in \mathscr{R}^{n_{s}}$, regulated output $y$, measurable outputs $\dot{q}_{s}, q_{m}, \dot{q}_{m}$ and smooth disturbance $y_{L}(t) \in \mathscr{L}_{\infty}$. Find conditions on $D, R, \mu, \eta$ such that, for all smooth desired output functions $y_{d}(t) \in \mathscr{L}_{\infty}$ with known derivative $\dot{y}_{d}(t) \in \mathscr{L}_{\infty}$, global torque tracking with internal stability is achieved, i.e. $\lim _{t \rightarrow \infty}\left(y-y_{d}\right)=0$ with all internal signals bounded. Further, for underactuated machines, we will require asymptotic flux amplitude tracking, that is, for a given bounded function $\beta(t) \geq \delta>0$, we must have that $\lim _{t \rightarrow \infty}\left|\left\|\lambda_{r}\right\|-\beta(t)\right|=0$.

\subsection{Design procedure}

The rationale of our design, takes off from the passive subsystems decomposition of Section 2 and, "disregarding" the mechanical dynamics, attempts to regulate the generated torque $y$ by imposing a desired value to the currents $\dot{q}_{e}$. There are therefore three natural steps to follow:

1. Apply the passive subsystems decomposition of Section 2 to the motor, to view $\Sigma_{e}$ as the "system to be controlled", and $\Sigma_{m}$ as a passive disturbance. To ensure 


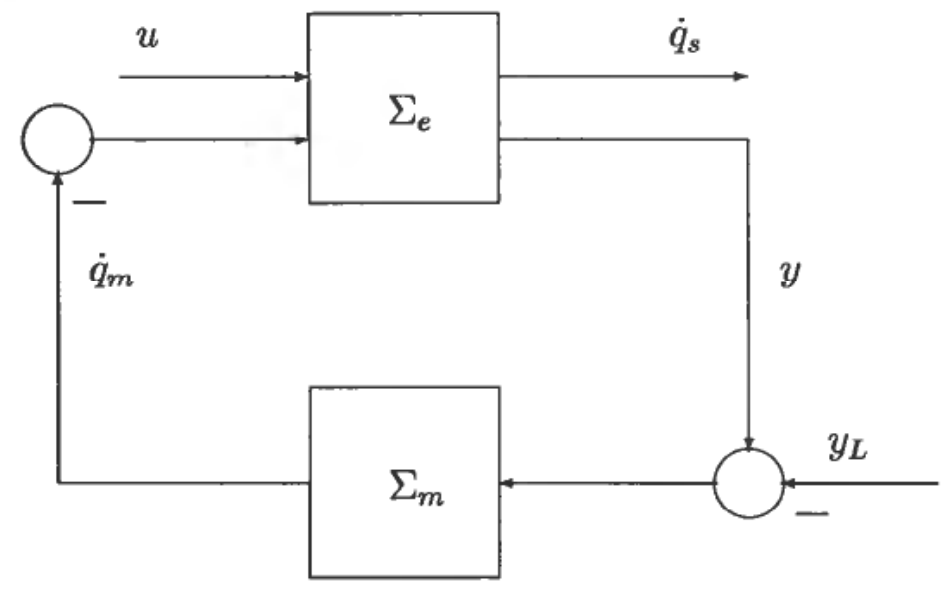

Figure 1. Passive subsystem decomposition.

the latter does not "destroy" the stability of the loop we must inject damping to $\Sigma_{e}$ so as to strengthen its passivity property to strict passivity.

2. Define a set of "attainable" currents $\dot{q}_{e d}$, i.e., those for which we can find a control law that ensures $\lim _{t \rightarrow \infty} \dot{q}_{e}=\dot{q}_{e d}$. To this end we shape the energy of the closed loop to match a desired energy (storage) function, which we choose here as

$$
H_{e d}:=\frac{1}{2} \dot{\tilde{q}}_{e}^{T} D_{e} \dot{\tilde{q}}_{e},
$$

where the current error is defined as

$$
\dot{\tilde{q}}_{e}:=\dot{q}_{e}-\dot{q}_{e d}
$$

3. Among the attainable currents choose $\dot{q}_{e d}$ to deliver the desired torque $y_{d}$, that is, such that if $\dot{q}_{e} \equiv \dot{q}_{e d}$ then $y \equiv y_{d}$. Finally, give conditions under which $\lim _{t \rightarrow \infty} y=y_{d}$ with internal stability.

\section{Strict passifiability via damping injection}

In this section we will carry out the first step of our design procedure, namely, decomposition of the model into passive subsystems $\Sigma_{e}, \Sigma_{m}$, and strict passifiability of $\Sigma_{e}$.

\subsection{Feedback decomposition}

Proposition 5.1. The system $\Sigma\left(D, R, M_{e}, \mu, \eta\right)$ can be represented as the negative feedback interconnection of two passive subsystems (see Fig. 1)

$$
\begin{gathered}
\Sigma_{e}: \mathscr{L}_{2 e}^{n_{s}+1} \rightarrow \mathscr{L}_{2 e}^{n_{s}+1}:\left[\begin{array}{c}
u \\
-\dot{q}_{m}
\end{array}\right] \mapsto\left[\begin{array}{c}
\dot{q}_{s} \\
y
\end{array}\right] \\
\Sigma_{m}: \mathscr{L}_{2 e} \rightarrow \mathscr{L}_{2 e}:\left(y-y_{L}\right) \mapsto \dot{q}_{m}
\end{gathered}
$$

\section{Proof}

The proof is a corollary of Lemma 2.1 noting that the Lagrangian of the electric machine (3.2) can be decomposed into

$$
\mathscr{L}_{e}\left(\dot{q}_{e}, q_{m}\right)=\frac{1}{2} \dot{q}_{e}^{T} D_{e} \dot{q}_{e}+\mu^{T} \dot{q}_{e}, \mathscr{L}_{m}\left(q_{m}, \dot{q}_{m}\right)=\frac{1}{2} D_{m} \dot{q}_{m}^{2}-\mathscr{V}\left(q_{m}\right)
$$




\subsection{Conditions for damping injection}

We now identify a class of machines for which-injecting damping to $\Sigma_{e}$-we can ensure that the map from control input to measurable output is output strictly passive. The physical interpretation of the assumptions is given after the proposition.

Proposition 5.2. Consider the subsystem $\Sigma_{e}\left(D_{e}, R_{e}, \mu, \eta\right)$. Assume

A.1. $R_{e}:=\operatorname{diag}\left\{R_{s}, R_{r}\right\}$ with $R_{s} \in \mathscr{R}^{n_{s} \times n_{s}}, R_{r} \in \mathscr{R}^{n_{r} \times n_{r}}$ diagonal matrices and $R_{r}>0$

A.2. The $n_{r} \times n_{r}$-dimensional $(2,2)$ block of the matrix

$$
W_{1}=\frac{d D_{e}}{d q_{m}}
$$

is zero, i.e.

$$
W_{1}=\left[\begin{array}{ll}
\left(W_{1}\right)_{11} & \left(W_{1}\right)_{12} \\
\left(W_{1}\right)_{21} & 0_{n_{r} \times n_{r}}
\end{array}\right]
$$

A.3. The non-actuated rotor components of the vector $\mu$ are independent of $q_{m}$, that is

$$
W_{2}=\frac{d \mu}{d q_{m}}:=\left[\begin{array}{c}
W_{2 s} \\
0
\end{array}\right], W_{2 s} \in \mathscr{R}^{n_{s}}
$$

Under these conditions there is a nonlinear output feedback of the form

$$
u=v+W_{2 s} \dot{q}_{m}-K_{1}\left(q_{m}, \dot{q}_{m}\right) \dot{q}_{s}
$$

such that the map $v \mapsto \dot{q}_{s}$ is output strictly passive for all $q_{m}, \dot{q}_{m} \in \mathscr{L}_{2 e}$.

\section{Proof}

The dynamics of $\Sigma_{e}$ is described by (3.4) which we repeat here for ease of reference

$$
D_{e} \ddot{q}_{e}+W_{1} \dot{q}_{m} \dot{q}_{e}+W_{2} \dot{q}_{m}+R_{e} \dot{q}_{e}=M_{e} u
$$

Closing the loop with (5.1) we get

$$
D_{e} \ddot{q}_{e}+C_{e}\left(q_{m}, \dot{q}_{m}\right) \dot{q}_{e}+R_{e s}\left(q_{m}, \dot{q}_{m}\right) \dot{q}_{e}=M_{e} v
$$

where we have defined

$$
C_{e}:=\frac{1}{2} W_{1} \dot{q}_{m}, R_{e s}:=R_{e}+\frac{1}{2} W_{1} \dot{q}_{m}+\left[\begin{array}{rr}
K_{1} & 0 \\
0 & 0
\end{array}\right]
$$

Taking the time derivative of the total energy of $\Sigma_{e}$, that is

$$
H_{e}:=\frac{1}{2} \dot{q}_{e}^{T} D_{e} \dot{q}_{e},
$$

along the trajectories of $\Sigma_{e}$ we get

$$
\dot{H}_{e}=\dot{q}_{s}^{T} v-\dot{q}_{e}^{T} R_{e s} \dot{q}_{e}
$$

Suppose that

$$
K_{1}=K_{1}^{T}>\sup _{q_{m}, q_{m}}\left\{\frac{\dot{q}_{m}^{2}}{4}\left(W_{1}\right)_{12} R_{r}^{-1}\left(W_{1}\right)_{12}^{T}-\frac{1}{2}\left(W_{1}\right)_{11} \dot{q}_{m}\right\}
$$

Then, by using standard matrix results, the symmetric matrix $R_{e s}$ can be shown to be 
uniformly positive definite in the sense that $\inf _{q m, q m} \lambda_{\min }\left\{R_{e s}\right\} \geq \alpha>0$, where $(\cdot)_{i j}$ denotes the $(i, j)$-th submatrix of a block matrix and $\lambda_{\min }\{\cdot\}$ is the minimum eigenvalue. Integration of $\dot{H}_{e}$ completes the proof.

\subsection{Remarks}

1. Notice that strict passivity is achieved, via the nonlinear damping term $K_{1} \dot{q}_{s}$, (with $K_{1}$ explicitly depending on $q_{m}$ and $\dot{q}_{m}$ ) which recovers the positivity of the "damping" matrix $R_{e s}$.

2. In the case of full actuation, i.e. $n_{s}=n_{e}$, the required positivity of $R_{e s}$ is guaranteed if

$$
K_{1}=K_{1}^{T}>-R_{e}-\frac{1}{2} W_{1} \dot{q}_{m}
$$

3. Assumption A.1. is a reasonable condition of damping of the nonactuated dynamics which is satisfied in all electric machines. The problem is with condition (5.4), which shows that to overcome the imprecise knowledge of the rotor resistances we will have to inject high gains into the loop.

4. A.2. is a decoupled dynamics condition equivalent to requiring that in the magnetic coenergy the contribution of the terms quadratic in $\dot{q}_{r}$ be independent of $q_{m}$. Physically, this translates into the condition that if there are rotor windings, then the rotor flux induced by the rotor currents must be independent of the rotor position. This means that the stator must have uniform reluctance properties (non-salient and of uniform magnetic material). This assumption is satisfied by many machines, e.g. classical Park [74] and poly-phase [72] machines.

5. Since the torque (3.6) consists of one component due to the currents, and the other of purely magnetical origin, and since we have no control on the magnetic fields from the permanent magnets, it is reasonable to expect that we must eliminate the effect on $\Sigma_{e}$ of the flux linkages due to the permanent magnets. This explains the need for assumption A.3. Physically, this assumption also means that if the machine has rotor windings, then the stator must have uniform reluctance properties i.e., if the machine has permanent magnets, then they can only be placed on the rotor. As can be seen from (5.1), the term from the permanent magnets must be cancelled out. The need for this cancellation is a drawback of the scheme. However, the term is generally a vector with periodic functions in a measurable quantity (position), and proportional to a constant which can be precisely identified.

\section{Current tracking via energy shaping}

We now define the "attainable" currents $\dot{q}_{e d}$ of the second step of the design procedure.

Proposition 6.1. If in (5.1) $v$ and $\dot{q}_{e d} \in \mathscr{L}_{\infty}^{n_{e}}$ satisfy

$$
M_{e} v=D_{e} \ddot{q}_{e d}+C_{e} \dot{q}_{e d}+R_{e s} \dot{q}_{e d}
$$

then (see (4.1) for the definition of the current error) $\dot{\tilde{q}}_{e} \rightarrow 0$ as $t \rightarrow \infty$ independently of $q_{m}, \dot{q}_{m}$ and the choice of $\dot{q}_{e d}$. Furthermore, when $\dot{q}_{e d}$ is bounded then $\dot{q}_{m}, \dot{q}_{e}$ and $y$ also bounded. In addition, boundedness of $\ddot{q}_{e d}$ ensures that $\ddot{q}_{e}$ and $v$ are also bounded. 


\section{Proof}

Rewriting (5.2) in terms of the error signals gives

$$
D_{e} \ddot{\tilde{q}}_{e}+C_{e} \dot{\tilde{q}}_{e}+R_{e s} \dot{\tilde{q}}_{e}+\psi
$$

with

$$
\psi:=M_{e} v-\left(D_{e} \ddot{q}_{e d}+C_{e} \ddot{q}_{e d}+R_{e s} \dot{q}_{e d}\right)
$$

Then (6.1) implies $\psi \equiv 0$, and the dynamics of the system is fully described by

$$
\begin{gathered}
D_{e} \ddot{\tilde{q}}_{e}+C_{e} \dot{\tilde{q}}_{e}+R_{e s} \dot{\tilde{q}}_{e}=0 \\
D_{m} \ddot{q}_{m}+R_{m} \dot{q}_{m}=y-y_{L}
\end{gathered}
$$

These equations are locally Lipschitz in state, and under the assumptions on the desired torque and load torque, they are continuous in $t$, so there exists $t_{1}>0$ such that in the time interval $\left[0, t_{1}\right)$ the solutions exists and are unique.

Taking the time derivative of the desired energy function

$$
H_{e d}=\frac{1}{2} \dot{\tilde{q}}_{e}^{T} D_{e} \dot{\tilde{q}}_{e}
$$

along the trajectories of (6.2) we get

$$
\dot{H}_{e d}=-\dot{\tilde{q}}_{e}^{T} R_{e s} \dot{\tilde{q}}_{e}, \forall t \in\left[0, t_{1}\right)
$$

It follows from (6.6) and the proof of Proposition 5.2 that

$$
\dot{H}_{e d}=-\dot{\tilde{q}}_{e}^{T} R_{e s} \dot{\tilde{q}}_{e} \leq-\alpha\left\|\dot{\tilde{q}}_{e}\right\|^{2}, \forall t \in\left[0, t_{1}\right)
$$

and we can conclude that

$$
\left\|\dot{\tilde{q}}_{e}(t)\right\| \leq m_{e}\left\|\dot{\tilde{q}}_{e}(0)\right\| \mathrm{e}^{-\rho_{e} t}, \forall t \in\left[0, t_{1}\right)
$$

holds with

and

$$
m_{e}=\sqrt{\frac{\lambda_{\max }\left(D_{e}\right)}{\lambda_{\min }\left(D_{e}\right)}}
$$

which are independent of $t_{1}$.

$$
\rho_{e}=\frac{\alpha}{\lambda_{\max }\left(D_{e}\right)}
$$

From this, and since $\dot{q}_{e d} \in \mathscr{L}_{\infty}^{n_{\infty}}$, we deduce that $\dot{q}_{e}$ is bounded on the open interval $\left[0, t_{1}\right)$. Now, we are going to prove that it remains bounded also on the closed interval $\left[0, t_{1}\right)$. To this end, notice that the right hand side of $(6.5)$ is also bounded on $\left[0, t_{1}\right)$, thus its solution cannot grow faster than an exponential, and consequently $\dot{q}_{m}, q_{m}$ remain bounded $\forall t \in\left[0, t_{1}\right]$. This in its turn ensures the boundedness of $R_{e s}$, and consequently $\left\|\dot{\tilde{q}}_{e}\right\|$ cannot escape to infinity in this time interval.

Since $m_{e}, \rho_{e}$ and $\alpha$ are independent of $t_{1}$, it is possible to repeat this argument for a new initial condition, to define solutions on the time interval $\left[t_{1}, 2 t_{1}\right]$. This shows that it is possible to extend this procedure to prove existence of solutions for the whole real axis, and the system cannot have finite escape time. It follows from (6.8) that

$$
\lim _{t \rightarrow \infty} \dot{\tilde{q}}_{e}=0
$$

and we can conclude that $\dot{q}_{e}$ is bounded, which implies that $y$ is also bounded. From this, and the definition of the load torque (3.3), it follows from $\dot{q}_{m}$ remains bounded. Now, it follows from (6.4) and (6.3) that $\ddot{q}_{e}$ and $v$ will be bounded if $\ddot{q}_{e d}$ is also bounded. 


\section{From current tracking to torque tracking}

It is now convenient to take a brief respite and recapitulate our derivations. The first step of Subsection 4.2 was carried out in Section 5 where we designed an inner control loop to ensure that (5.2) defines a strictly passive mapping $v \mapsto \dot{q}_{s}$. In Section 6 we carried out the second step, that is, we established a relationship between the control signal $v$ and $\dot{q}_{e d}(6.1)$ which implies $\lim _{t \rightarrow \infty} \dot{q}_{e}=\dot{q}_{e d}$. The third step, to which this section is devoted, demands the definition of $\dot{q}_{e d}$ from the "attainable" set that delivers the desired torque and the establishment of conditions under which current tracking implies torque tracking. Notice that these steps are not straightforward since $D_{e}, C_{e}$ and $R_{e s}$ (6.1), and $y$ (3.6), depend on $q_{m}$ and $\dot{q}_{m}$, thus some additional conditions on the couplings between the subsystems must be satisfied. These conditions are expressed in terms of restrictions on the parameters $D_{e}, R_{e}, \mu, \eta$ of the general electric machine model.

\subsection{Desired current behaviour}

Motivated by (3.6) we proposed to define $\dot{q}_{e d}$ such that, for a given desired torque $y_{d}$ the equation

$$
y_{d}=\frac{1}{2} \dot{q}_{e d}^{T} W_{1} \dot{q}_{e d}+W_{2} \dot{q}_{e d}+\eta
$$

holds. This gives

$$
y-y_{d}=\frac{1}{2} \dot{\tilde{q}}_{e}^{T} W_{1} \dot{\tilde{q}}_{e}+\dot{\tilde{q}}_{e}^{T} W_{1} \dot{q}_{e d}+W_{2}^{T} \dot{\tilde{q}}_{e}
$$

with the error signal $\dot{\tilde{q}}_{e}:=\dot{q}_{e}-\dot{q}_{e d}$. Since $W_{1}$ and $W_{2}$ are bounded, it follows that asymptotic torque tracking will be achieved if we can ensure $\lim _{t \rightarrow \infty} \dot{q}_{e d}$ with $\dot{q}_{e d} \in \mathscr{L}_{\infty}^{n_{e}}$.

- It is clear then that to attain the torque tracking objective we must define bounded $\dot{q}_{e d}$ and $v$ such that (7.1) and (6.1) both hold.

Towards this end, first notice that in the case of fully actuated machines $M_{e}=\mathscr{I}_{n e}$ and there are no restrictions on the set of "attainable" currents. That is, in this case, for any given $\ddot{q}_{e d}, \dot{q}_{e d}$, we can make $\psi \equiv 0$ by a suitable selection of $v$.

Now, to treat the case of underactuated machines, where we do not have enough control actions to directly set $\psi \equiv 0$ for any given $\dot{q}_{e d}$, we find it convenient to partition $\psi=\left[\psi_{s}^{T}, \psi_{r}^{T}\right]^{T}$ with $\psi_{s} \in \mathscr{R}^{n_{s}}$. Since $\psi_{s} \equiv 0$ can always be solved with a suitable choice of $v$, (see the definition of $M_{e}$ ), we concentrate our attention on the solution of $\psi_{r} \equiv 0$. We will show in the sequel that, although (6.1) and (7.1) are intimately related, additional restrictions on the machine model are required for a simultaneous solution. Interestingly enough, it turns out that these "decoupling" conditions are (a stronger version of) the well known Blondel-Park (BP) transformation conditions which are fundamental in the analysis of rotating machines [41], [47].

\subsection{Decoupling conditions}

The following definition is in order:

Definition 7.1. We say the machine $\Sigma\left(D, R, M_{e}, \mu, \eta\right)$ is $B P$ transformable if there exists a current transformation

$$
\dot{z}_{e}=P\left(q_{m}\right) \dot{q}_{e}=P_{1} \mathbf{e}^{-U q_{m}} \dot{q}_{e}
$$


such that the dynamics of $\Sigma_{e}$ in these coordinates are independent of $q_{m}$ (but still depend on $\dot{q}_{m}$ ). $P_{1}$ is any nonsingular constant matrix. If furthermore the matrix $U$ is of the form

$$
U=\left[\begin{array}{cc}
0 & 0 \\
0 & U_{22}
\end{array}\right]=-U^{T}=\left[\begin{array}{cc}
0 & 0 \\
0 & -U_{22}^{-1}
\end{array}\right]
$$

then we say the machine is strongly $B P$ transformable.

From the structure of the matrix $U$ above we can see the strong BP transformability means that the decoupling is achieved rotating only the rotor variables. As will become clear later, this condition is needed when the rotor circuits are not actuated, as in the induction motor case.

In the fundamental paper [41] necessary and sufficient conditions for BP transformability are given. Since our definition of the BP transformation slightly differs from the one given in [41], and for the sake of self-containment, we give below a simplified version of their theorem, whose proof is given in Appendix A.

Proposition 7.1. If there exists a constant matrix $U \in \mathscr{R}^{n_{e} \times n_{e}}$ solution of

$$
\begin{aligned}
U D_{e}-D_{e} U & =W_{1} \\
R_{e} U & =U R_{e} \\
U W_{2} & =\frac{d W_{2}}{d q_{m}}
\end{aligned}
$$

then $\Sigma\left(D, R, m_{e}, \mu, \eta\right)$ is BP transformable. In this case the dynamics of $\Sigma_{e}$ (see 3.4)) in the coordinates $\dot{z}_{e}$ is described by

$$
D_{e}(0) P_{1}^{-1} \ddot{z}_{e}+U D_{e}(0) P_{1}^{-1} \dot{q}_{m} \dot{z}_{e}+W_{2}(0) \dot{q}_{m}+R_{e} P_{1}^{-1} \dot{z}_{e}=\mathbf{e}^{-U q_{m}} M_{e} u=M_{e} u^{\prime}
$$

while the dynamics of $\Sigma_{m}$ (see (3.5)) is described by

$$
\begin{aligned}
D_{m} \ddot{q}_{m}+R_{m} \dot{q}_{m} & =y-y_{L} \\
y & =\dot{z}_{e}^{T} P_{1}^{-T} U D_{e}(0) P_{1}^{-1} \dot{z}_{e}+W_{2}^{T}(0) P_{1}^{-1} \dot{z}_{e}+\eta
\end{aligned}
$$

\section{Example 7.1. Park's transformation}

For a class of synchronous machines with inductance matrix as in (3.9), the BP transformation to the $d q 0$-frame is given as [33]

$$
P\left(q_{m}\right)=\frac{2}{3}\left[\begin{array}{ccc}
\cos \left(n_{p} q_{m}\right) & \cos \left(n_{p} q_{m}-\frac{2 \pi}{3}\right) & \cos \left(n_{p} q_{m}+\frac{2 \pi}{3}\right) \\
\sin \left(n_{p} q_{m}\right) & \sin \left(n_{p} q_{m}-\frac{2 \pi}{3}\right) & \sin \left(n_{p} q_{m}+\frac{2 \pi}{3}\right) \\
\frac{1}{2} & \frac{1}{2} & \frac{1}{2}
\end{array}\right]
$$

This transformation can also be written as

$$
P\left(q_{m}\right)=P_{1} \mathbf{e}^{-U q_{m}}
$$

where

$$
U=\frac{n_{p}}{\sqrt{3}}\left[\begin{array}{ccc}
0 & -1 & 1 \\
1 & 0 & -1 \\
-1 & 1 & 0
\end{array}\right] \quad P_{1}=\frac{1}{3}\left[\begin{array}{ccc}
2 & -1 & -1 \\
0 & -\sqrt{3} & \sqrt{3} \\
1 & 1 & 1
\end{array}\right]
$$


and $U$ satisfies (7.3)-(7.5). The inverse transformation is given as

$$
P^{-1}\left(q_{m}\right)=\mathbf{e}^{U q_{m}} P_{1}^{-1}=\left[\begin{array}{ccc}
\cos \left(n_{p} q_{m}\right) & \sin \left(n_{p} q_{m}\right) & 1 \\
\cos \left(n_{p} q_{m}-\frac{2 \pi}{3}\right) & \sin \left(n_{p} q_{m}-\frac{2 \pi}{3}\right) & 1 \\
\cos \left(n_{p} q_{m}+\frac{2 \pi}{3}\right) & \sin \left(n_{p} q_{m}+\frac{2 \pi}{3}\right) & 1
\end{array}\right]
$$

Several slightly different forms are encountered in the literature, [19], [23]. This stems from the choice of the matrix $P_{1}$, which is sometimes chosen from a power preserving objective, or with the objective of making $P_{1}$ orthogonal.

\subsection{Remarks}

1. For the purpose of the present paper, the key feature of BP transformable machines is that the components of torque that also involves currents become independent of rotor position (see (7.8)) when expressed in suitable coordinates. Notice also that, for constant speed, the electrical subsystem in (7.6) is linear time invariant when $u^{\prime}$ is taken as the new input. This fundamental property has been exploited in the literature to determine stability properties in stationary operation [70].

2. The underlying fundamental assumption for the machine to be BP transformable, is that the windings are sinusoidally distributed [74], giving a sinusoidal air-gap magnetomotive force (MMF) and sinusoidally varying elements in the inductance matrix $D_{e}$. For a practical machine, this means that the first harmonic in a Fourier approximation of the MMF must give a sufficiently close approximation of the real MMF. Examples of machines in which higher order harmonics must be taken into account, are the squarewave brushless DC motors in [48], and machines with significant saliency in the air gap [65].

3. It is interesting to remark that the BP transformation cannot be derived from a canonical transformation [21] $z=z(q)$ of the generalized coordinates and momenta. This fact is presented in Appendix B.

4. Since the matrix $U$ is real and skew-symmetric, it follows that $\mathbf{e}^{-U q_{m}}$ is an orthogonal transformation, and the transformation $P\left(q_{m}\right)$ is bounded.

\section{Main results}

We now proceed to relate the property of BP transformability with our problem of definition of desired currents $\dot{q}_{e d}$. This is carried out in the following subsections, where we treat underactuated $\left(n_{s}<n_{e}\right)$ and fully actuated machines $\left(n_{s}=n_{e}\right)$ separately.

\subsection{Underactuated machines, $n_{s}<n_{e}$}

Proposition 8.1. Assume the machine $\Sigma\left(D, R, M_{e}, \mu, \eta\right)$ is strongly $B P$ transformable, satisfies $\mu=\eta \equiv 0$, the $(2,1)$ block of $D_{e}$ is nonsingular, and that $\beta(t)$ is a bounded strictly positive twice differentiable function with bounded first and second order derivatives. Under these conditions, the following definition of $\dot{q}_{\text {ed }}$ satisfies (6.1) and (7.1) for any given $y_{d}$ 


$$
\dot{q}_{e d}=\left[\begin{array}{c}
\dot{q}_{s d} \\
\dot{q}_{r d}
\end{array}\right]=\left[\begin{array}{c}
\left(D_{e}\right)_{21}^{-1}\left[\mathscr{I}_{n_{r}}+\left(D_{e}\right)_{22}\left\{\frac{1}{\beta^{2}} y_{d} U_{22}^{-1}+\frac{\dot{\beta}}{\beta} R_{r}^{-1}\right\}\right] \lambda_{r d} \\
-\left[\frac{1}{\beta^{2}} y_{d} U_{22}^{-1}+\frac{\dot{\beta}}{\beta} R_{r}^{-1}\right] \lambda_{r d}
\end{array}\right]
$$

where $\lambda_{r d}$ is the solution of the differential equation

$$
\dot{\lambda}_{r d}=\frac{1}{\beta^{2}(t)} y_{d} R_{r} U_{22}^{-1} \lambda_{r d}+\frac{\dot{\beta}(t)}{\beta(t)} \lambda_{r d}
$$

with initial conditions such that $\left\|\lambda_{r d}(0)\right\|=\beta(0)$. Furthermore,

$$
\left\|\lambda_{r d}(t)\right\|=\beta(t), \forall t \geq 0
$$

\section{Proof}

The last statement of the proposition follows immediately by taking the time derivative of

$$
\frac{1}{2}\left\|\lambda_{r d}\right\|^{2}
$$

substituting (8.2), and using the fact that strong BP transformability implies $R_{r} U_{22}^{-1}+\left(R_{r} U_{22}^{-1}\right)^{T}=0$.

To simplify the notation of the rest of the proof we find it convenient to introduce the desired flux $\lambda_{d}=\left[\lambda_{s d}^{T}, \lambda_{r d}^{T}\right]^{T}$ as

$$
\lambda_{d}:=D_{e} \dot{q}_{e d}
$$

Some simple calculations using (6.3), (5.3) and (8.3) show that ${ }^{8}$

$$
\psi_{r} \equiv 0 \Leftrightarrow \dot{\lambda}_{r d}+R_{r} \dot{q}_{r d}=0
$$
(7.3))

Now, notice that BP transformability of the machine allows us to write (7.1) as (see

$$
y_{d}=\dot{q}_{e d}^{T} U D_{e} \dot{q}_{e d}
$$

which, in terms of the desired fluxes and currents looks like

$$
y_{d}=\dot{q}_{e d}^{T} U \lambda_{d}
$$

If further the machine is strongly $B P$ transformable then

$$
\begin{aligned}
y_{d} & =\dot{q}_{r d}^{T} U_{22} \lambda_{r d} \\
& =-\dot{\lambda}_{r d}^{T} R_{r}^{-1} U_{22} \lambda_{r d}
\end{aligned}
$$

where we have set $\psi_{r} \equiv 0$ and used (8.4) in the last equation. From this we see that for (7.1) to hold, we must define $\dot{\lambda}_{r d}$ such that (8.5) always holds. It is straightforward to verify that this is the case when $\dot{\lambda}_{r d}$ is defined as in (8.2), using $U_{22}^{-T}=-U_{22}^{-1}$ (see Definition 7.1), the symmetry of $R_{r}$ and the fact that $\left\|\lambda_{r d}(t)\right\|=\beta(t)$.

The proof is completed using (8.4) to obtain $\dot{q}_{r d}$ and the definition of $\lambda_{d}$ to calculate $\dot{q}_{s d}$.

Our main result for underactuated machines is contained in the theorem below.

Theorem 8.1. Consider the machine model (3.4), (3.5), (3.6). Assume that the machine

${ }^{8}$ See (3.1) and the first remark in Subsection 3.2 for the physical motivation behind this choice of relations between desired fluxes and currents. 
is strongly BP transformable (Definition 7.1), $\mu=0, \eta=0,\left(D_{e}\right)_{21}$ is nonsingular and A.1.-A.2. of Proposition 5.2. hold. Under these conditions, there exists a dynamic output feedback controller that ensures global asymptotic torque tracking with internal stability. Furthermore, for all $\beta(t)$ (bounded strictly positive twice differentiable with bounded first and second order derivatives), we have $\lim _{t \rightarrow \infty}||\left|\lambda_{r} \|-\beta(t)\right|=0$.

Proof

The control law is obtained from (5.1) and (6.3), setting $\psi_{s} \equiv 0$. To this end, we use the definition of $\dot{q}_{e d}$ of Proposition 8.1. Notice that $\ddot{q}_{e d}$ is bounded, and can be computed from the available measurements provided $\dot{y}_{d}$ is known.

Convergence of $\dot{\tilde{q}} \rightarrow 0$ follows from the arguments of Section 6 . Boundedness of $\dot{q}_{e d}$ follows from (8.1) and the boundedness of $\lambda_{r d}$ and $y_{d .}$. This establishes asymptotic torque tracking.

Electrical rotor flux tracking is a consequence of the convergence of the currents to their desired values and the chosen norm of $\lambda_{r d}$, since

$$
\lambda_{r d}-\lambda_{r}=\left(D_{e}\right)_{21}\left(\dot{q}_{s d}-\dot{q}_{s}\right)+\left(D_{e}\right)_{22}\left(\dot{q}_{r d}-\dot{q}_{r}\right)
$$

and $D_{e}\left(q_{m}\right)$ is bounded with respect to $q_{m}$.

\subsection{Fully actuated machines, $n_{s}=n_{e}$}

For fully actuated machines $M_{e}=\mathscr{I}_{n_{e}}$, and as previously explained, we can make $\psi=0$ by a suitable selection of $v$ for given $\dot{q}_{e d}$ and $\ddot{q}_{e d}$. The main difficulty is to find $\dot{q}_{\text {ed }}$ such that (7.1) is satisfied. This is done by choosing the desired currents from the BP transformed torque equation, since the matrices relating the transformed currents and the torque are not dependent on $q_{m}$, which considerably simplifies the choice.

Theorem 8.1. Consider the machine model (3.4), (3.5), (3.6). Assume the machine is BP transformable (Definition 7.1), and A.1.-A.3. of Proposition 5.2. hold. Under these conditions, if the desired currents and their derivatives are defined as

$$
\begin{aligned}
& \dot{q}_{e d}=\mathbf{e}^{U q_{m}} P_{l}^{-1} \dot{z}_{e d} \\
& \ddot{q}_{e d}=U \mathbf{e}^{U q_{m}} P_{1}^{-1} \dot{q}_{m} \dot{z}_{e d}+\mathbf{e}^{U q_{m}} P_{1}^{-1} \ddot{z}_{e d}
\end{aligned}
$$

where $\dot{z}_{e d}$ is chosen to satisfy

$$
y_{d}-\eta=\dot{z}_{e d}^{T} P_{1}^{-T} U D_{e}(0) P_{1}^{-1} \dot{z}_{e d}+W_{2}^{T}(0) P_{1}^{-1} \dot{z}_{e d}
$$

with $\dot{z}_{e d}, \ddot{z}_{e d} \in \mathscr{L}_{\infty}^{n_{e}}$, and the dynamic output feedback controller defined in (5.1), with

$$
v=D_{e} \ddot{q}_{e d}+C_{e} \dot{q}_{e d}+R_{e s} \dot{q}_{e d}
$$

ensures global asymptotic torque tracking with internal stability.

\section{Proof}

The expression for the torque in the transformed system is, according to (7.7)

$y-\eta=\dot{z}_{e}^{T} P_{1}^{-T} U D_{e}(0) P$
Setting $\dot{\tilde{z}}_{e}=\dot{z}_{e}-\dot{z}_{e d}$ and using (8.8) gives

$$
y-\eta=\dot{z}_{e}^{T} P_{1}^{-T} U D_{e}(0) P_{1}^{-1} \dot{z}_{e}+W_{2}^{T}(0) P_{1}^{-1} \dot{z}_{e}
$$

$$
y-y_{d}=\dot{\tilde{z}}_{e}^{T} P_{1}^{-T} U D_{e}(0) P_{1}^{-1} \dot{\tilde{z}}_{e}+2 \dot{\tilde{z}}_{e}^{T} P_{1}^{-T} U D_{e}(0) P^{-1} \dot{z}_{e d}+W_{2}^{T}(0) P_{1}^{-1} \dot{z}_{e}
$$

Since $\dot{\tilde{z}}_{e}=P\left(q_{m}\right) \dot{\tilde{q}}_{e}$, and $P\left(q_{m}\right)$ is a bounded transformation, it follows that

$$
\lim _{t \rightarrow \infty} \dot{\tilde{q}}_{e}=0 \quad \stackrel{\leftrightarrow}{\Downarrow} \lim _{t \rightarrow \infty} \dot{\tilde{z}}_{e}=0
$$

It is clear that $\dot{z}_{e d} \in \mathscr{L}_{\infty}^{n_{e}} \Leftrightarrow \dot{q}_{e d} \in \mathscr{L}_{\infty}^{n_{e}}$, and $\ddot{z}_{e d} \in \mathscr{L}_{\infty}^{n_{e}}, \dot{q}_{m} \in \mathscr{L}_{\infty} \Rightarrow \ddot{q}_{e d} \in \mathscr{L}_{\infty}^{n_{e}}$. Defining $v$ as in (8.9) gives $\psi=0$, and the arguments of Section 6 hold. 


\subsection{Remarks}

1. Notice that the assumption in Proposition 8.1. that the $(2,1)$ block of $D_{e}$ is nonsingular implies that the number of actuated and nonactuated windings must be equal. This is the case for typical induction motors, where this matrix is a nonsingular rotation matrix.

2. Eq. (8.2) has a solution of the form

$$
\lambda_{r d}(t)=\frac{\beta(t)}{\beta(0)} \mathbf{e}\left(U_{22}\right)^{-1} \rho_{d}(t) \lambda_{r d}(0), \dot{\rho}_{d}(t)=\frac{r_{r}}{\beta^{2}(t)} y_{d}(t), \rho_{d}(0)=0
$$

This gives an interpretation of the desired flux in terms of its rotation angle, whose speed is related to the desired torque.

3. During the derivation of the model and controller, we have assumed the use of a voltage source inverter for the input to the actuated windings. If the inverter used is a current source inverter or a fast current control is used, it follows that the inputs to the actuated windings will be the currents $\dot{q}_{s} \equiv \dot{q}_{s d}$, where $\dot{q}_{s d}$ is the vector of desired currents for the actuated windings, as defined in the previous sections.

4. The quadratic form in (8.8) is in general not easy to solve for the components of $\dot{z}_{\text {ed }}$. Examples of solutions for certain motors are given in Section 9.

5 . For $3 \phi$ machines the currents of the transformed system are usually denoted with subscripts $d, q$ and 0 . For machines in which the symmetrical windings has an isolated neutral, the zero sequence of the transformed currents is exactly zero, which defines a natural choice for desired value of this current.

6. The desired transformed currents could be chosen from a similar objective as in the field oriented approach. The transformed torque equation is generally given as

$$
y=c\left\{\lambda_{d} \dot{z}_{q}-\lambda_{q} \dot{z}_{d}\right\}
$$

where $c$ is a constant, and $\lambda_{d}, \lambda_{q}$ are $d$ and $q$ components of the transformed flux vector. If we can make $\lambda_{q} \dot{z}_{d}$ equal to zero and $\lambda_{d}$ constant, it will be possible to control the torque by specifying $\dot{z}_{q}$.

\section{Examples}

\subsection{Two-phase model of uniform air gap squirrel cage induction motors}

In this section the controller (5.1), (6.1) with desired currents from (8.1)-(8.2), will be applied to the two-phase $\alpha \beta$ model of an $n_{p}$ pole pair squirrel cage machine with uniform air gap. This model could be interpreted as an equivalent model of the usual $3 \phi$ machine, or stemming from a reduction of phases in the more general polyphase machine through transformations like those presented in [71]. It is common practice to substitute the (single)-cage rotor which has a uniform conductor distribution with a fictitious sinusoidally wound rotor (poles and phases corresponding to stator windings) for analytical purposes. This implies that only the first harmonic of the rotor MMF is modelled, but this is not detrimental for the subsequent analysis and controller design. However, care should be taken in cases of double-cage or deep bar rotors [71], [69].

The model has $n_{e}=4, n_{s}=n_{r}=2$, and its parameters are

$$
\begin{aligned}
D_{e}\left(q_{m}\right) & =\left[\begin{array}{cc}
L_{s} \mathscr{I}_{2} & L_{s r} \mathbf{e}^{\mathscr{I n}_{p} \mathscr{q}_{m}} \\
L_{s r} \mathbf{e}^{-\mathscr{I}_{p} q_{m}} & L_{r} \mathscr{I}_{2}
\end{array}\right], \mu=0, \eta=0 \\
R_{e} & =\left[\begin{array}{cc}
R_{s} \mathscr{I}_{2} & 0 \\
0 & R_{r} \mathscr{I}_{2}
\end{array}\right], M_{e}=\mathscr{I}_{2}
\end{aligned}
$$


where $L_{s}, L_{s r}, L_{r}$ are inductance parameters, $R_{r}, R_{s}$ are resistances, and

$$
\mathscr{J}=\left[\begin{array}{cc}
0 & -1 \\
1 & 0
\end{array}\right], \mathbf{e}^{\mathscr{f} n_{p} q_{m}}=\left[\begin{array}{cc}
\cos \left(n_{p} q_{m}\right) & -\sin \left(n_{p} q_{m}\right) \\
\sin \left(n_{p} q_{m}\right) & \cos \left(n_{p} q_{m}\right)
\end{array}\right]
$$

Equations (7.3)-(7.5) are satisfied for

$$
U_{22}=-n_{p} \mathscr{J}=n_{p}\left[\begin{array}{cc}
0 & 1 \\
-1 & 0
\end{array}\right]
$$

and $\mathrm{e}^{y_{n} q_{m}}$ is the BP transformation of the rotor quantities from the rotor reference system to the stator reference system. This gives the desired rotor flux (see (8.2) and (8.11))

$$
\lambda_{r d}=\mathbf{e}^{\rho_{d} \mathscr{F}}\left[\begin{array}{c}
\beta(t) \\
0
\end{array}\right], \dot{\rho}_{d}=\frac{R_{r}}{n_{p} \beta^{2}(t)} y_{d}(t), \rho_{d}(0)=0
$$

where the rotation angle (relative to the rotor system) is defined by the familiar relation between desired slip, $\dot{\rho}_{d}$ and desired torque $y_{d}$.

The choice of desired currents then follows from (8.1), and the input to the stator windings is given by

$$
\begin{aligned}
& u=v-K_{1}\left(\dot{q}_{m}\right) \dot{q}_{s} \\
& v=L_{s} \ddot{q}_{s d}+L_{s r} \mathbf{e}^{\mathscr{f} n_{p} q_{m}} \ddot{q}_{r d}+L_{s r} n_{p} \mathscr{J} \mathbf{e}^{\mathscr{f} n_{p} q_{m}} \dot{q}_{m} \dot{q}_{r d}+R_{s} \dot{q}_{s d}+K_{1}\left(\dot{q}_{m}\right) \dot{q}_{s d}
\end{aligned}
$$

where

$$
K_{1}\left(\dot{q}_{m}\right)=\left(\frac{n_{p}^{2} L_{s r}^{2}}{4 \varepsilon} \dot{q}_{m}^{2}+k_{1}\right) \mathscr{I}_{2}, 0<\varepsilon<\min \left\{R_{s}, R_{r}\right\}, k_{1} \geq 0
$$

has been chosen to satisfy (5.4).

The application of this observerless control scheme was first proposed in [16], and its extension to speed and position control is presented in [56].

It is important to underscore that in [56] it has been shown that under the simplifying assumptions of constant speed and that the inverter can be modelled as a current source, the passivity-based control reduces exactly to the well known indirect field oriented control (FOC). For a voltage source inverter, the difference from indirect FOC is the additional nonlinear damping term introduced in the input. Furthermore, in [73] it is shown that the global stability properties are preserved for all estimates $\hat{T}_{r}$ of rotor time constant

$$
T_{r}=\frac{L_{r}}{R_{r}}
$$

such that

$$
\frac{1}{2} T_{r}<\hat{T}_{r}<\infty,
$$

which is a condition that will be satisfied in most practical applications.

\subsection{Synchronous motors}

In the last years, synchronous motors, and especially permanent magnet motors have become an attractive alternative to induction motors in the low to medium power range [6]. These machines are generally more expensive than induction motors ${ }^{9}$, but

\footnotetext{
${ }^{9}$ The difference in price will be reduced as low price high-energy permanent magnets become available.
} 
have higher efficiency due to the fact that the rotor losses are negligible. They also have reduced size compared to the induction motors.

The controller as given by (5.1), (8.9) with currents satisfying (8.8), can be applied to this type of motor as follows.

Using the transformation given in (7.9)-(7.10), and the model given in (3.9)-(3.10), the torque can be expressed in new coordinates $\dot{z}_{e}=\left[\dot{z}_{d}, \dot{z}_{q}, \dot{z}_{0}\right]^{T}$ as

$$
y=\frac{3 n_{p}}{2}\left\{\left(L_{d}-L_{q}\right) \dot{\mathbf{z}}_{d} \dot{z}_{q}+\lambda_{m} \dot{z}_{q}\right\}
$$

where

$$
L_{d}=L_{l s}+\frac{3}{2}\left(L_{A}+L_{B}\right), L_{q}=L_{l s}+\frac{3}{2}\left(L_{A}-L_{B}\right) .
$$

The desired currents are chosen as

$$
\dot{z}_{e d}=\left[\begin{array}{c}
0 \\
2 y_{d} \\
3 n_{p} \lambda_{m} \\
0
\end{array}\right]
$$

from which it follows that $\dot{z}_{e d}, \ddot{z}_{e d} \in \mathscr{L}_{\infty}^{3}$, whenever $y_{d}, \dot{y}_{d} \in \mathscr{L}_{\infty}$.

To satisfy (5.5), taking the uncertainty of the resistances into account, we choose $K_{1}$ as

$$
K_{1}=-\frac{1}{2} W_{1} \dot{q}_{m}+k \mathscr{I}_{3}, k>0
$$

The input is then given from (8.9) and (5.1).

It must be pointed out that if $L_{B} \neq 0$, the airgap is nonuniform. According to Remark 5.3. our method can be used for such machines as long as there are no rotor windings, which is the case here.

The above approach can also be extended to synchronous reluctance motors ${ }^{10}$ with the same inductance matrix as in (3.9). In these machines there are no permanent magnets or windings in the rotor, hence $\lambda_{m}=0$ and the torque is given as [22]

$$
y=\frac{3 n_{p}}{2}\left(L_{d}-L_{q}\right) \dot{z}_{d} \dot{z}_{q}
$$

from which it follows that one of the desired currents should be constant, and the other proportional to desired torque.

Also, if the synchronous machine has a field winding on the rotor instead of permanent magnets, $\lambda_{m}$ will be proportional to the current in the field winding, which is usually chosen to be constant or varying according to a field weakening objective. The choice of the other desired currents could be done as previously explained.

\subsection{PM stepper motor}

As a last example, we show in this section how to apply the proposed controller (5.1), (8.9) with currents satisfying (8.8), to a PM stepper motor.

With the model given in (3.11)-(3.12), the transformation to the $d q$-frame is [41]

$$
P\left(q_{m}\right)=\mathbf{e}^{-U q_{m}}=\left[\begin{array}{cc}
\cos \left(N_{r} q_{m}\right) & \sin \left(N_{r} q_{m}\right) \\
-\sin \left(N_{r} q_{m}\right) & \cos \left(N_{r} q_{m}\right)
\end{array}\right], U=\left[\begin{array}{cc}
0 & -N_{r} \\
N_{r} & 0
\end{array}\right]
$$

\footnotetext{
${ }^{10}$ This motor has been proposed as an alternative to other AC-machines, see [40].
} 
where $U$ satisfies (7.3)-(7.5). This transformation is orthogonal, and $P^{-1}\left(q_{m}\right)=P^{T}\left(q_{m}\right)$.

The torque expressed in new coordinates $\dot{z}_{e}=\left[\dot{z}_{d}, \dot{z}_{q}\right]^{T}$ is

$$
y=K_{m} \dot{z}_{q}-K_{D} \sin \left(4 N_{r} q_{m}\right)
$$

and we choose the desired currents as

$$
\dot{z}_{e d}=\left[\begin{array}{c}
0 \\
\frac{1}{K_{m}}\left\{y_{d}+K_{D} \sin \left(4 N_{r} q_{m}\right)\right\}
\end{array}\right]
$$

Notice that (5.5) is satisfied for the choice $K_{1}=k \mathscr{I}_{2}>0$, where $k>0$, and $y_{d} \in \mathscr{L}_{\infty}$ implies $\dot{z}_{e d} \in \mathscr{L}_{\infty}^{2}$, and boundedness of $\ddot{q}_{e d}$ follows from the boundedness of $\dot{y}_{d}$ and $\dot{q}_{m}$. The input is then given from (8.9) and (5.1).

As previously pointed out, the underlying assumption of BP-transferability is the sinusoidally distribution of the MMF. It can be discussed whether this is a good approximation in the case of stepper motors, with concentrated windings, significant air gap saliency and often hybrid rotor construction. The BP-transform above has however been used in several applications, and we have adopted this convention here.

Its worth to point out that the controller in [4] can be obtained from the passivity based approach if it is applied to the full system, without dividing the system into electrical and mechanical parts as done here.

\section{Experimental results}

To illustrate the performance of the proposed method, we implemented the scheme in Sub-section 9.1. to control speed/position of a 4-pole $3 \phi$ squirrel cage induction motor with $\Delta$-connected stator phases from the German company Lust GmbH. We give here only a brief description of the equipment and the experiments and refer the reader to [51] for further details.

The equivalent parameters ${ }^{11}$ are:

\begin{tabular}{llc}
\hline \multicolumn{3}{l}{ Nominal parameters of motor system } \\
\hline$R_{s}$ & Stator resistance & $1.9 \Omega$ \\
$R_{r}$ & Rotor resistance & $3 \cdot 0 \Omega$ \\
$L_{s}$ & Stator inductance & $0 \cdot 130 \mathrm{H}$ \\
$L_{r}$ & Rotor inductance & $0 \cdot 130 \mathrm{H}$ \\
$L_{s r}$ & Mutual inductance & $0 \cdot 120 \mathrm{H}$ \\
$n_{p}$ & Number of pole pairs & 2 \\
$\dot{q}_{m N}$ & Rated speed & $3000 \mathrm{rpm}$ \\
$\beta_{N}$ & Rated rotor flux amplitude & $0 \cdot 2 \mathrm{~Wb}$ \\
$D_{m}$ & Inertia (rotor and load) & $5 \cdot 53 \mathrm{kgcm}$ \\
$y^{N}$ & Nominal torque & $1.5 \mathrm{Nm}$ \\
$P_{\text {rated }}$ & Rated power & $400 \mathrm{~W}$ \\
$u_{D C}$ & DC-link voltage of inverter & $300 \mathrm{~V}$ \\
$I_{M A X}$ & Maximum line currents & $4.5 \mathrm{~A}$ \\
\hline
\end{tabular}

\footnotetext{
${ }^{11}$ Identification of all $2 \phi$ motor parameters was carried out by the manufacturer on sample motors in the same production series. They were given in the motors data sheet with $\pm(5-10) \%$ accuracy.
} 
Position was measured with an incremental encoder and a quadruple counter, giving a position resolution of

$$
\frac{2 \pi}{4 \cdot 4096} \approx 4 \cdot 0 \cdot 10^{-4} \mathrm{rad} \text {. }
$$

Speed was estimated from position measurements using a backward difference approximation, and computed at one third of the main sampling frequency to increase resolution. A dSPACE controller board with a TMS320C31 main processor and a TMS320P14 slave processor were used for controller implementation. Two of the line currents were measured using LEM transducers, and converted to digital signals using 12 bit A/D converters. The controller was first implemented in SIMULINK, and then converted to C-code, compiled and downloaded to the board using dSPACE software. Symmetric carrier-based PWM was implemented on the slave processor. The switching pattern is calculated at a frequency of $10 \mathrm{kHz}$ for the three phases, from voltage references transferred from the main processor at the end of each sampling interval. For the discretization a simple first order Euler approximation was used, with a sampling time of $T_{\text {sampl }}=300 \mu \mathrm{s}$. The combination of this sampling time with the number of encoder lines and backward difference estimation, gave a speed resolution of

$$
\frac{1}{4.4096} /\left(3 T_{\text {sampl }}\right) \cdot 60 \approx 4 \cdot 1 \mathrm{rpm}
$$

(See [42]) for speed or position tracking, the desired torque was chosen as in [56], with an extra integral term to compensate for the unknown load torque (friction in bearings of motor and inertial load), giving

$$
\begin{aligned}
y_{d} & =D_{m} \ddot{q}_{m d}-z-f\left(q_{m}-q_{m d}\right)+\hat{y}_{L} \\
\dot{z} & =-a z+b\left(\dot{q}_{m}-\dot{q}_{m d}\right), a, b>0, z(0)=\dot{q}_{m}(0)-\dot{q}_{m d}(0) \\
\dot{\hat{y}_{L}} & =-\gamma e, \gamma \geq 0
\end{aligned}
$$

where $q_{m d}, \dot{q}_{m d}$ are the rotor position and speed reference, and $f \equiv 0$ for speed tracking. The error term in the integral action was set to $e=q_{m}-q_{m d}$ for position tracking, and $e=\dot{q}_{m}-\dot{q}_{m d}$ in the case of speed tracking. The references were generated by linear first order filtering of step/square-wave signals from implementations of the SIMULINK signal generator. Higher order derivatives of references for position/speed and flux amplitude $\beta(t)$ were obtained from state space representations of linear filters.

The only true way of verifying flux tracking is to compare measured flux with its reference. Unfortunately, flux measurement is far from trivial, and hence not implemented in the experimental setup. We have therefore used the current model observer from [5] to estimate flux amplitude $\hat{\lambda}_{d}$

$$
\begin{aligned}
& \hat{\lambda}_{d}=\frac{L_{s r}}{T_{r} p+1} \hat{i}_{d}, \quad p:=\frac{d}{d t} \\
& \hat{i}_{d}=\cos \left(\theta_{a}\right) i_{\alpha}+\sin \left(\theta_{a}\right) i_{\beta} \\
& \hat{i}_{q}=-\sin \left(\theta_{a}\right) i_{\alpha}+\cos \left(\theta_{a}\right) i \beta \\
& \theta_{a}=n_{p} q_{m}+\int_{0}^{t} \frac{L_{s r}}{T_{r}} \frac{\hat{i}_{q}}{\hat{\lambda}_{d}} d t
\end{aligned}
$$

where $T_{r}=L_{r} / R_{r}$ and $i_{\alpha}, i_{\beta}$ are stator currents of the equivalent $2 \phi$ model. These were calculated from the $3 \phi$ line current measurements $I_{A}$ and $I_{B}$. Due to the singularity in the flux speed calculation, it was necessary to substitute $\hat{\lambda}_{d}$ in the division above by a small constant $c=0.001$ whenever $\hat{\lambda}_{d} \leq c$. This estimator was run in parallel with the controller, and not used for feedback purposes. 

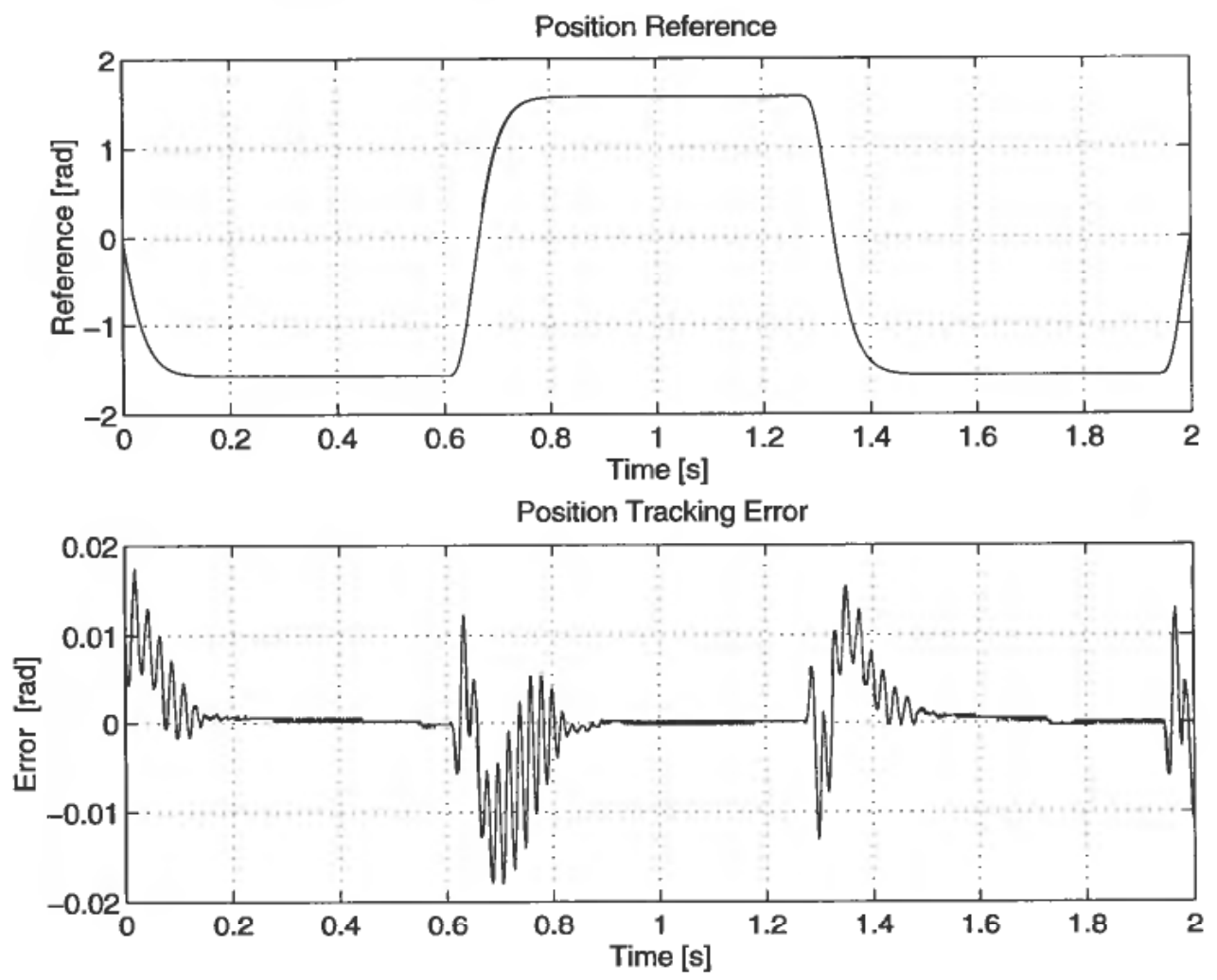

Figure 2. Position reference $q_{m d}(t)$ (upper figure) and tracking error $\left(q_{m}-q_{m d}\right)$.

A sampling time of $300 \mu$ s was high enough to allow for simultaneously logging of several signals, without significant response deterioration. By running only the code strictly necessary for the implementation (no observer, only logging of execution time), the sampling time could be set to $T=147 \mu \mathrm{s}$.

The following parameters were used for position tracking: $a=1000, b=95, f=41$, $\gamma=70$ : For speed and flux tracking we used $a=1000, b=320, f=0, \gamma=3.85$.

Figure 2 shows position reference and tracking error for a $180^{\circ}$ turn of the motor. As can be seen from the lower plot the maximum error is approximately $1^{\circ}$. The next figure shows that precision of position control was only restricted by the resolution of the position measurement. The digital jittering could be removed by using a deadzone in the position controller. Due to position dependent friction, controller parameters had to be set low enough to avoid oscillatory behaviour at certain "worst-case" positions. In the lower plot of Fig. 3 the flux error during position tracking for a constant reference of $\beta=0.2 \mathrm{~Wb}$ is shown. Perfect control can hardly be expected with parameters taken directly from the data sheet and a simple discretization, and there is a maximum error of $0.009 \mathrm{~Wb}$ during transients. Reference voltages for PWM and measured line currents for two of the three phases are shown in Fig. 4. Voltage references were below the theoretical limits for the PWM ( $\pm 150 \mathrm{~V})$, and the line currents did not saturate. This was also the case for all of the experiments reported here, but for space limitations we do not include more than one plot of currents and voltages.

In Figs. 5 and 6 we have shown examples of flux tracking and speed regulation with 

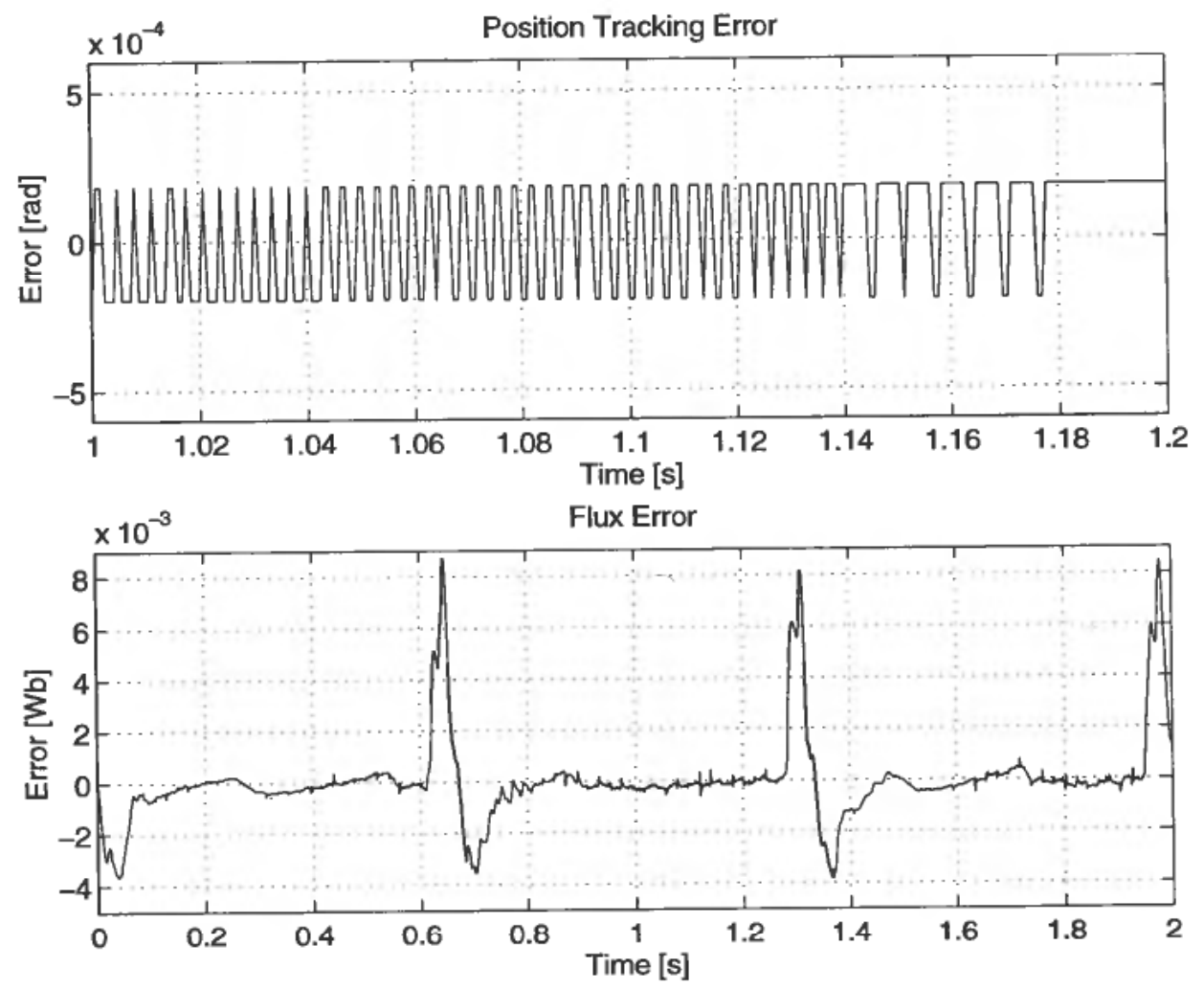

Figure 3. Window of position tracking error and flux error $\left(\hat{\lambda}_{d}-\beta\right)$.

and without the term $\dot{\beta}(t)$ in the controller. Comparison shows that this term is necessary for high quality flux tracking. Finally, in Fig. 7 an example of speed tracking with a reference close to a step, and constant $\beta=0.2 \mathrm{~Wb}$ is shown. The speed follows its reference closely, and results in only minor peaks in flux during transients, due to unmodelled effects.

The parameter tuning was done online while observing responses, and the scheme was easy to tune, as compared to implementations of other observer-based FOC schemes.

Further experimental results from the application of this controller can be found in [15] (speed tracking and flux regulation), [29] (comparison to feedback linearizing controllers) and [51] (position, speed and flux tracking with comparison to FOC).

\section{Concluding remarks}

We have studied in this paper the output feedback global tracking problem for the generalized electric machine's model. A passivity-based method is used to design the controller which proceeded in three steps. First, the machine is decomposed as the feedback interconnection of two passive subsystems - electrical and mechanical. Then, a nonlinear damping is injected to make the electrical subsystem strictly passive. Finally, an energy-shaping controller is designed to make the currents converge exponentially to a desired value that delivers the required torque. Our main contribution is the establishment of physically interpretable conditions on the model such that the method can be successfully applied. To further relax these conditions, it is our belief that we must combine passivity ideas with the powerful new dynamic extension 

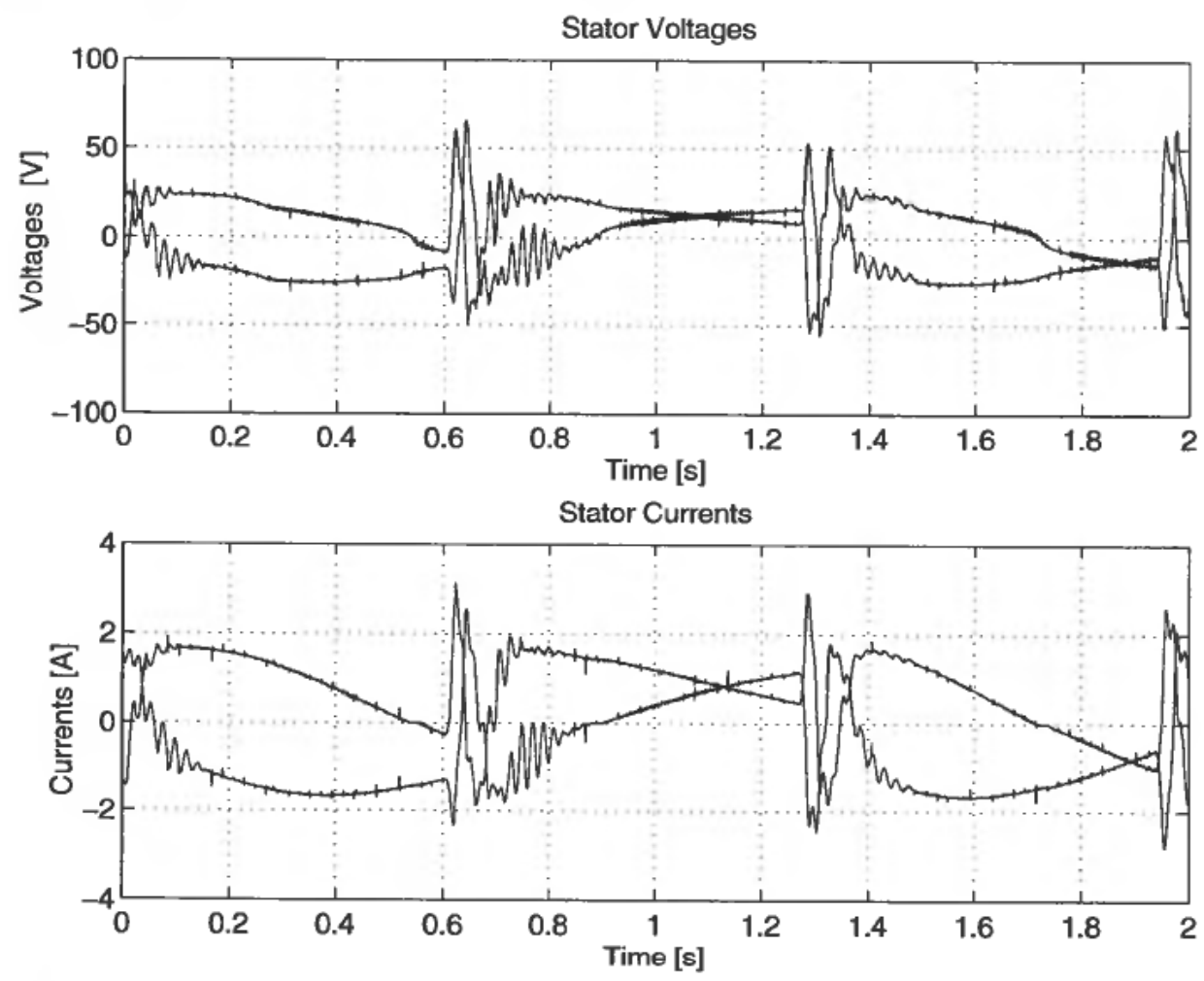

Figure 4. $3 \phi$ stator phase voltage references $u_{a}, u_{b}$ and measured line currents $I_{A}, I_{B}$.

techniques for stabilization of nonlinear systems. Some research along these lines for the robotics problem has been reported in [8].

The passivity-based approach gives control schemes which provides global stability results for the resulting system. Further, there is no need for observers since unmeasurable states are not used, and there are no singularities, hence obviating the need for special precautions to be taken at for example start-up. The performance of the scheme, as measured with the exponential convergence rate of desired currents (and consequently outputs) to their desired values, can be explicitly derived for each machine using the results in Sections 5 and 6. It follows that the rate of convergence is restricted by the time constant of the unactuated windings. This is a consequence of the fact that additional damping cannot be injected into this dynamics, since the involved states are unmeasurable. As discussed in [29] the same limitation applies to feedback linearizing schemes, where the restriction is now imposed on the observer convergence.

To establish the relationship of our controller to existing schemes we notice that our control signal consists of a nonlinear damping term added to the reference dynamics. Henceforth, following [53], it can be classified as an indirect vector control scheme, which is the most widely used implementation of field oriented control, (especially well suited for operation close to zero speed [42]). In particular, for the speed control of the induction motor, it is shown in [56] that the passivity-based controller exactly reduces to indirect field oriented control under some simplifying assumptions, namely constant speed and current fed inverter, (for which the additional problem of stator dynamics is not present). Also, this connection to indirect FOC shows that under the same 

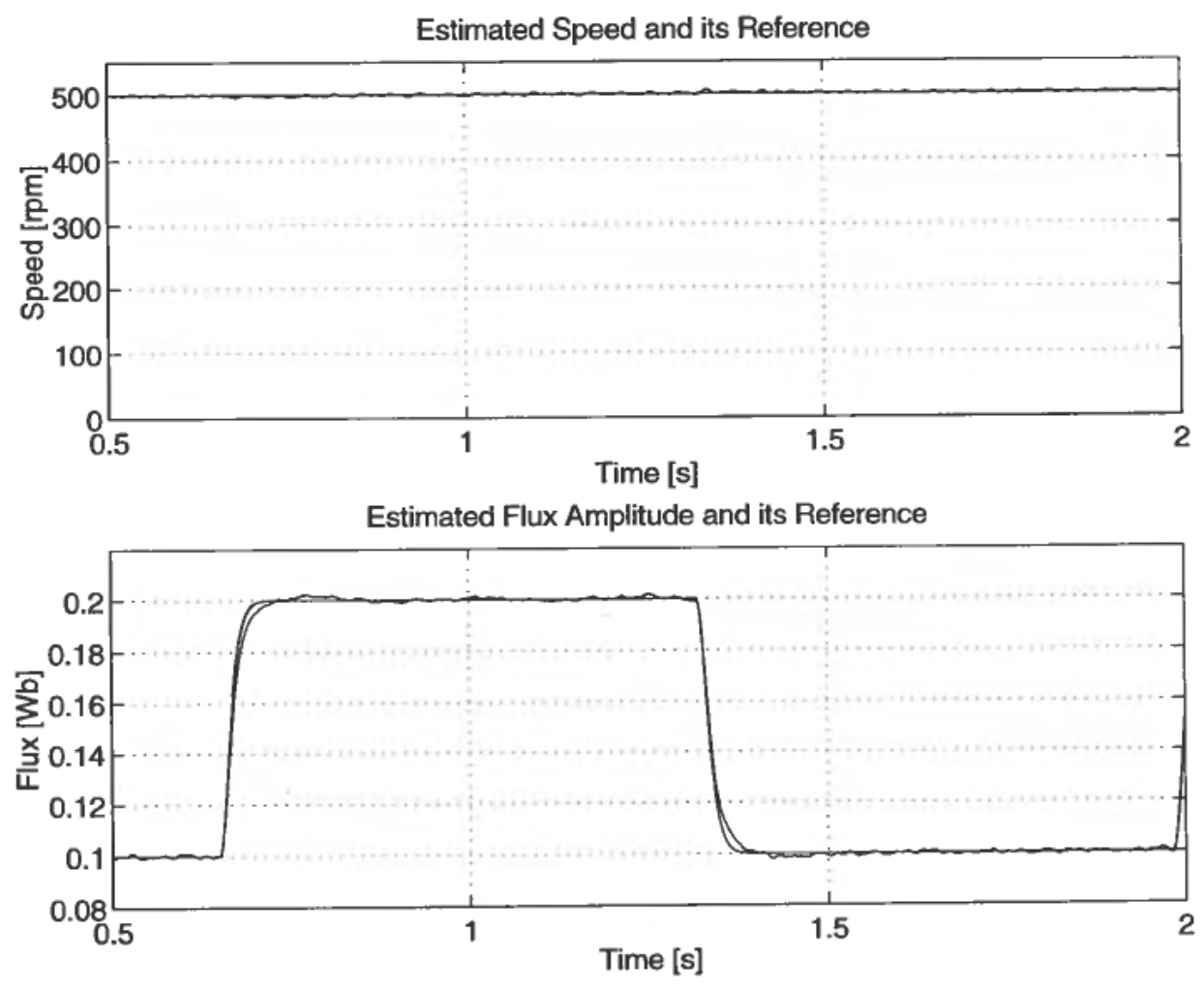

Figure 5. Example of flux tracking. Speed and its reference in upper figure. Flux amplitude and its reference in lower figure.

assumptions, the computational burden of the passivity based approach is no greater than in this implementation of FOC.

In practice, the assumptions of constant and known parameters will not hold. For instance resistances will vary due to temperature changes and the skin effect at high frequencies, and inductances will change when magnetic saturation occurs. To assess the sensitivity of the stability of indirect field oriented control vis a vis these assumptions we carried out in [73] a robustness analysis. In that paper we proved that stability is preserved despite large variations in rotor resistance and inductance. In view of the downward compatibility mentioned above, these robustness properties are inherited by the passivity based controller. It may be possible to enhance performance by identifying parameters on-line. Globally stable adaptive controllers have been reported in [14], unfortunately requiring full state measurement. The relaxation of this assumption, together with extension to the case of nonlinear magnetics, use of speed observers, and friction compensation are the main topics for our future research in this field. Some interesting work on rotor resistance adaptation may be found in [12].

Since the passivity based approach exploits the systems energy properties, which are invariant under a change of coordinates, this gives the possibility of controller implementation in a general $d q$-frame, chosen from the objectives of minimizing computational burden and increasing numerical robustness.

Experimental results from the application of the proposed controller to an induction motor have been included. Even if an ad hoc discretization and a speed estimation 


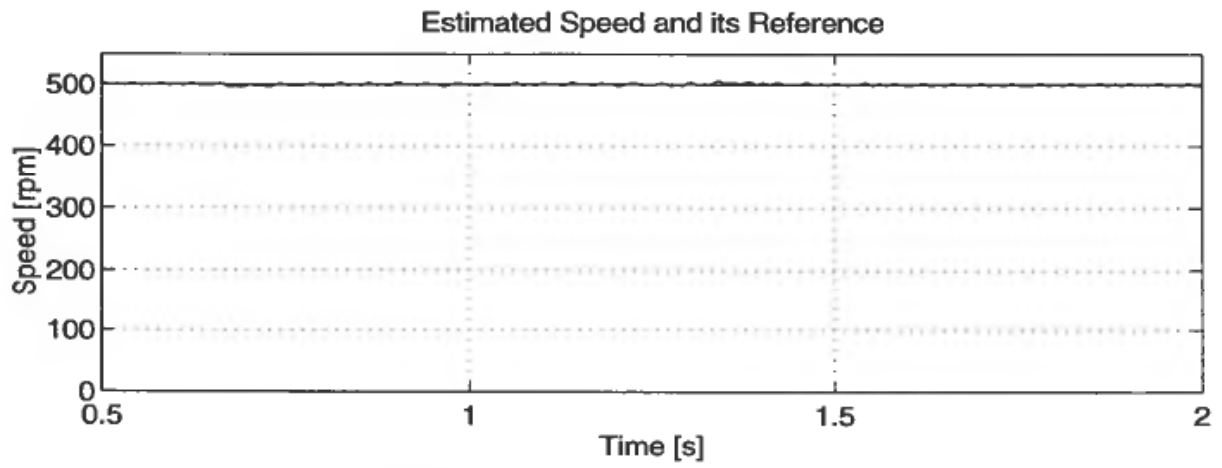

Estimated Flux Amplitude and its Reference

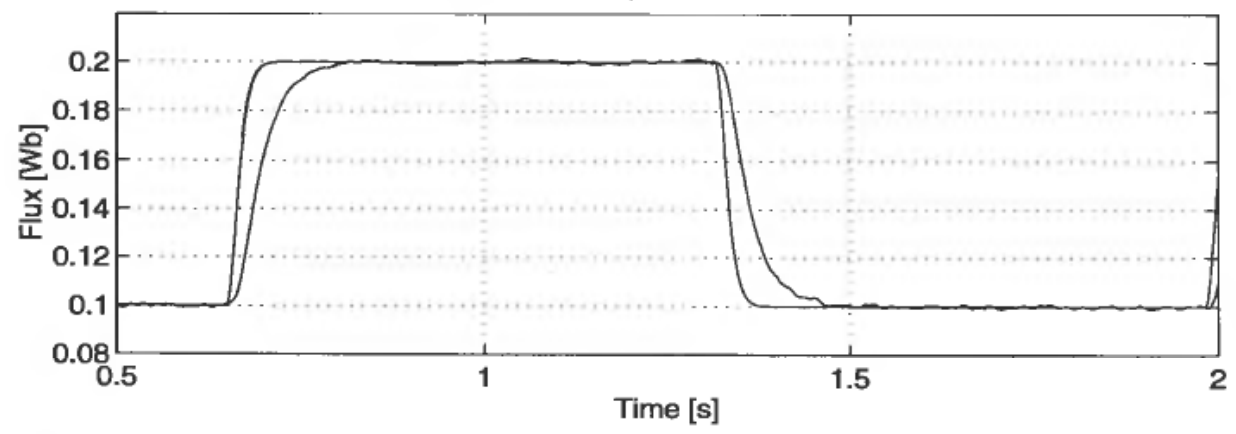

Figure 6. Example of flux tracking. Same as previous figure, but without $\dot{\beta}(t)$ in controller.
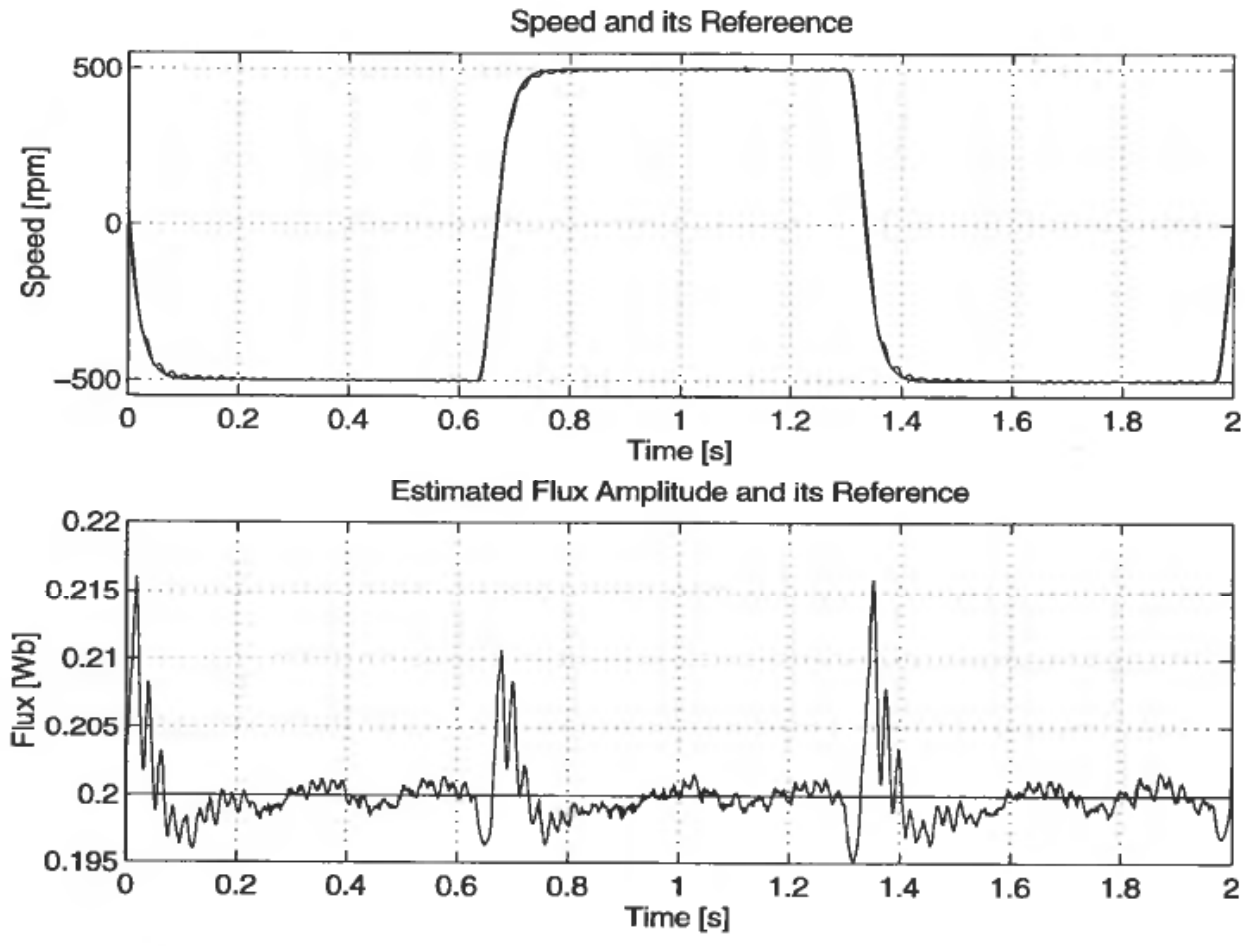

Figure 7. Example of speed tracking with constant flux reference. 
scheme not considered in the theoretical analysis have been used, high-performance position/speed and flux tracking was experienced. As expected from a scheme with global stability results, controller tuning was an easy task.

As pointed out in [65], there is a potential advantage in working with a general model instead of specializing the model equations, and we hope that the results in this paper will motivate for additional research in the area of servo applications utilizing a general machine's model and its passivity properties. One such problem is tracking control of a robot manipulator with AC-drives. In a recent paper [57] we have provided an answer to this question by extending the results in [10] to the global case and the use of a general BP transformable machine.

\section{ACKNOWLEDGEMENTS}

The main parts of this work were carried out while the first author was visiting Université de Technologie de Compiègne. The hospitality of this institution is gratefully acknowledged.

\section{Appendices}

\section{A. Proof of Proposition 7.1.}

For the proof of Proposition 7.1, we need the following lemma:

Lemma A.1.

$$
\frac{d D_{e}\left(q_{m}\right)}{d q_{m}} \neq 0 \Rightarrow U+U^{T}=0
$$

\section{Proof}

Note that the differential equation (7.3) has the unique solution $D_{e}\left(q_{m}\right)=\mathbf{e}^{U q_{m}} D_{e}(0) \mathbf{e}^{-U q_{m}}[28]$.

$$
\begin{aligned}
D_{e}=D_{e}^{T} & \Rightarrow \mathbf{e}^{U q_{m}} D_{e}(0) \mathbf{e}^{-U q_{m}}=\mathbf{e}^{-U^{T} q_{m}} D_{e}(0) \mathbf{e}^{U T_{q_{m}}} \\
& \Rightarrow \mathbf{e}^{U^{T} q_{m}} \mathbf{e}^{U q_{m}} D_{e}(0)=D_{e}(0) \mathbf{e}^{U T_{q_{m}}} \mathbf{e}^{U q_{m}} \\
& \Rightarrow \mathbf{e}^{U q^{T} q_{m}} \mathbf{e}^{U} q_{m}=\mathscr{I} \\
& \Rightarrow U^{T}+U=0
\end{aligned}
$$

The third implication follows from the fact that $U$ and $D_{e}$ do not commute, unless

$$
\frac{d D_{e}\left(q_{m}\right)}{d q_{m}}=0(7.3)
$$

and this implies that $f(U)=\mathbf{e}^{U T q_{m}} \mathbf{e}^{U q_{m}}$ and $D_{e}$ cannot commute, unless $f(U) \equiv \mathscr{I}$.

\section{Proof of Proposition 7.1.}

From (7.2) it follows that

$$
\begin{aligned}
& \dot{q}_{e}=\mathbf{e}^{U q_{m}} P_{1}^{-1} \dot{z}_{e} \\
& \ddot{q}_{e}=\mathbf{e}^{U q_{m}} P_{1}^{-1} \ddot{z}_{e}+U \mathbf{e}^{U q_{m}} P_{1}^{-1} \dot{q}_{m} \dot{z}_{e}
\end{aligned}
$$

After inserting these two equations into (7.6), and multiplying from the left by $\mathbf{e}^{-U q_{m}}$, we get

$$
\begin{aligned}
\mathbf{e}^{-U q_{m}} D_{e}\left(q_{m}\right) \mathbf{e}^{U q_{m}} P_{1}^{-1} \ddot{z}_{e} & +\mathbf{e}^{-U q_{m}} D_{e}\left(q_{m}\right) U \mathbf{e}^{U q_{m}} P_{1}^{-1} \dot{q}_{m} \dot{z}_{e} \\
& +\mathbf{e}^{-U q_{m}} W_{1}\left(q_{m}\right) \dot{q}_{m} \mathbf{e}^{U q_{m}} P_{1}^{-1} \dot{z}_{e}+\mathbf{e}^{-U q_{m}} \mathrm{~W}_{2}\left(q_{m}\right) \dot{q}_{m} \\
& +\mathbf{e}^{-U q_{m}} R_{e} \mathbf{e}^{U q_{m}} P_{1}^{-1} \dot{z}_{\mathrm{e}}=\mathbf{e}^{-U q_{m}} M_{e} u
\end{aligned}
$$


From $D_{e}\left(q_{m}\right)=\mathbf{e}^{U q_{m}} D_{e}(0) \mathbf{e}^{-U q_{m}}$, and since (7.4) implies that $\mathbf{e}^{U q_{m}} R_{e}=R_{e} \mathbf{e}^{U q_{m}}[38]$, we note that

$$
\begin{aligned}
\mathbf{e}^{-U q_{m}} D_{e}\left(q_{m}\right) \mathbf{e}^{U q_{m}} & =D_{e}(0) \\
\mathbf{e}^{-U q_{m}} D_{e}\left(q_{m}\right) U \mathbf{e}^{U q_{m}} & =D_{e}(0) U \\
\mathbf{e}^{-U q_{m}} W_{1}\left(q_{m}\right) \mathbf{e}^{U q_{m}}(7.3) & =\mathbf{e}^{-U q_{m}}\left[U \mathbf{e}^{U q_{m}} D_{e}(0) \mathbf{e}^{-U q_{m}}-\mathbf{e}^{U q_{m}} D_{e}(0) U \mathbf{e}^{-U q_{m}}\right] \\
& =U D_{e}(0)-D_{e}(0) U \\
\mathbf{e}^{-U q_{m}} R_{e} \mathbf{e}^{U q_{m}} & =R_{e}
\end{aligned}
$$

In addition, it follows from (7.5) that $W_{2}\left(q_{m}\right)=\mathbf{e}^{U q_{m}} W_{2}(0)$. This implies that $\mathbf{e}^{-U q_{m}} W_{2}\left(q_{m}\right)=W_{2}(0)$, which is constant with respect to $q_{m}$. Finally, inserting (A.5-A.8) into (A.4), gives

$$
D_{e}(0) P_{1}^{-1} \ddot{z}_{e}+U D_{e}(0) P_{1}^{-1} \dot{q}_{m} \dot{z}_{e}+W_{2}(0) \dot{q}_{m}+R_{e} P_{1}^{-1} \dot{z}_{e}=\mathbf{e}^{-U q_{m}} M_{e} u
$$

For the transformed mechanical system $\Sigma_{m}$, we have the relations

$$
\begin{gathered}
\dot{q}_{e}^{T} W_{1}\left(q_{m}\right) \dot{q}_{e}=\dot{z}_{e}^{T} P_{1}^{-T} \mathbf{e}^{U T} q_{m} W_{1}\left(q_{m}\right) \mathbf{e}^{U q_{m}} P_{1}^{-1} \dot{z}_{\mathrm{e}} \\
\stackrel{\text { (A.1) }}{=} \dot{z}_{e}^{T} P_{1}^{-T} \mathbf{e}^{-U q_{m}} W_{1}\left(q_{m}\right) \mathbf{e}^{U q_{m}} P_{1}^{-1} \dot{z}_{e} \\
\stackrel{\text { (A.7) }}{=} \dot{z}_{e}^{T} P_{1}^{-T}\left[U D_{e}(0)-D_{e}(0) U\right] P_{1}^{-1} \dot{z}_{e} \\
=2 z_{e}^{T} P_{1}^{-T} U D_{e}(0) P_{1}^{-1} \dot{z}_{e}=2 \dot{z}_{e}^{T} P_{1}^{-T} D_{e}(0) U^{T} P_{1}^{-1} \dot{z}_{e} \\
W_{2}^{T}\left(q_{m}\right) \dot{q}_{e}=W_{2}^{T}\left(q_{m}\right) \mathbf{e}^{U q_{m}} P_{1}^{-1} \dot{z}_{e} \\
=W_{2}^{T}(0) P_{1}^{-1} \dot{z}_{e}
\end{gathered}
$$

B. Lemma on the BP transformation

Lemma B.l. Unless $U \equiv 0$, the velocities $\dot{z}=\left[\dot{z}_{e}^{T}, \dot{q}_{m}\right]^{T}$ introduced by the BP transformation cannot be derived from a transformation $z=Z(q)$ of the generalized coordinates $q=\left[q_{e}^{T}, q_{m}\right]^{T}$.

\section{Proof}

The transformation from the generalized electrical velocities $\dot{q}_{e}$ and the generalized mechanical velocity $\dot{q}_{m}$ to $\dot{z}=\left[\dot{z}_{e}^{T}, \dot{q}_{m}\right] T$ is

$$
\left[\begin{array}{c}
\dot{z}_{e} \\
\dot{q}_{m}
\end{array}\right]=\left[\begin{array}{cc}
P_{1} \mathbf{e}^{-} U q_{m} & 0 \\
0 & 1
\end{array}\right]\left[\begin{array}{c}
\dot{q}_{e} \\
\dot{q}_{m}
\end{array}\right]
$$

If $z=Z(q)$, then we must have

$$
\frac{\partial Z}{\partial q}=\left[\begin{array}{cc}
P_{1} \mathbf{e}^{-U q_{m}} & 0 \\
0 & 1
\end{array}\right]
$$

since

$$
\dot{z}=\frac{\partial Z}{\partial q} \dot{q} .
$$

From this, we find that $z_{e}$ must be of the form

$$
z_{e}=Z_{e}(q)=P_{1} \mathbf{e}^{-U q_{m}} q_{e}+c, c \in \mathscr{R}^{n_{e}}
$$

Taking the total time derivative gives

$$
\dot{z}_{e}=\frac{\partial Z_{e}}{\partial q} \dot{q}=-P_{1} \mathbf{e}^{-U q_{m}} U \dot{q}_{m} q_{e}+P_{1} \mathbf{e}^{-U q_{m}} \dot{q}_{e}
$$


from which it follows that since the BP transformation (see (7.2)) is defined as $\dot{z}_{e}=P_{1} \mathbf{e}^{-U q_{m}} \dot{q}_{e}$ we must have

$$
-P_{1} \mathbf{e}^{-U q_{m}} U \dot{q}_{m} q_{e}=0, \forall q_{e}
$$

For this to hold, $U$ must be the zero matrix, since $P_{1} \mathrm{e}^{-U q_{m}}$ is nonsingular, and consequently for $U \neq 0$ there is no transformation $z_{e}=Z_{e}(q)$ such that

$$
\dot{z}_{e}=\frac{\partial Z_{e}}{\partial q} \dot{q}
$$

\section{REFERENCES}

[1] AILON, A. and Ortega, R. (1993). An observer-based set-point controller for robot manipulators with flexible joints, System \& Control Letters, 21, 329-335.

ARNOLD, V. I. (1989). Mathematical Methods of Classical Mechanics, 2nd. ed. (SpringerVerlag).

[2] BeRGHUIS H. and NiJMEIJER, H. (1993). A passivity approach to controller-observer design for robots, IEEE Trans. Rob. Aut., 9, 740-754.

[3] Bodson, M., Chiasson, J. N., Novotnak. R. T. and Rekowski, R. B. (1993). High-Performance Nonlinear Feedback Control of a Permanent Magnet Stepper Motor, IEEE Trans. Cont. Syst. Tech., 1, 5-14.

[4] BoDSON, M., CHIASSON, J. and NovOTNAK, R. (1994). High-Performance Induction Motor Control Via Input-Output Linearization, IEEE Control Systems Magazine, 14, 25-33. BoSE, B. K. (1993). Power Electronics and Motion Control-Technology Status and Recent Trends, IEEE Trans. Indust. Appl., 29, 902-909.

[5] BOYD, S. and BARRAT, C. (1991). Linear controller design: Limits of performance, Prentice Hall, NY.

[6] Brogliato, R., Ortega, R. and Lozano, R. (1995). Globally Stable Nonlinear Controllers for Flexible Joint Manipulators: A Comparative Study, Automatics, 31, 941-956.

[7] Byrnes, C., Isidori, A. and Willems, J. C. (1991). Passivity, Feedback Equivalence and the Global Stabilization of Minimum Phase Nonlinear Systems, IEEE Trans Aut. Cont., 36, 1228-1240.

[8] CANUdAs de Wit, C., ORTega, R. and Seleme S. I. JR., (1993). Robot Motion Control using Induction Motor Drives, Proc. IEEE ICRA, Atlanta, Georgia, 533-538.

[9] CRANDAll, S. H. (Ed.) (1968). Dynamics of Mechanical and Electromechanical Systems (McGraw-Hill).

[10] Dawson, D., Hu, J. and Vedagarbha, P. (1995). An adaptive controller for a class of induction motor systems, Proc IEEE CDC, New Orleans, LA, USA, Dec. 13-15, $1567-1572$.

[11] Desoer, C. and Vidyasagar, M. (1975) Feedback Systems: Input-Output Properties (Academic Press).

[12] Espinosa-PÉReZ, G. (1993) Nonlinear Control of Induction Motors, Ph.D. Thesis, UNAM, Mexico.

[13] Espinosa-Pérez, G., Campos-Cantón, I., Lara-Reyes, P. and Gomez-Becerril, D. (1995). Passivity-based Speed Control of a $2 \phi$ Induction Motor: Experimental Results, Proc. IV IEEE International Power Electronics Congress, San Luis Potosí, Mexico, October 16-19.

[14] Espinosa-PéreZ, G. and OrTegA, R. (1994). State Observers are Unnecessary for Induction Motor Control, Systems \& Control Letters, 23, 315-323.

[15] Espinosa-Pérez, G. and OrTega, R. (1995). An Output Feedback Globally Stable Controller for Induction Motors, IEEE Trans. Aut. Cont., 40, 138-143.

[16] Espinosa-Pérez, G., Nicklasson, P. J. and Ortega, R. (1995) Control of Induction Motors in the Field Weakening Region, Proc. 34th IEEE CDC, New Orleans, LA, 2151-2152. FitZGERAld, A. E., KingsLeY, C. JR. and UMANS, S. D. (1983). Electric Machinery (McGraw-Hill).

[17] GÖKDERE, L. U. (1996). Passivity-based methods for control of induction motors, Ph.D. Thesis, University of Pittsburgh.

[18] Goldstein, H. (1980). Classical Mechanics, 2nd ed. (Addison-Wesley). 
[19] Hemati, N. (1994). Non-Dimensionalization of the Equations of Motion for PermanentMagnet Machines, Electric Machines and Power Systems, 23, 541-556.

[20] Hemati, N. and Leu, M. C. (1992). A Complete Model Characterization of Brushless dc Motors, IEEE Trans. Indust. Appl., 28, 172-180.

[21] IsIDORI, A. (1995). Nonlinear Control Systems, 3rd ed. (Springer-Verlag).

[22] ISIDORI, A. and BYRNES, C. (1990). Output regulation of nonlinear systems, IEEE Trans. Aut. Cont., 35, 131-140.

[23] JIANG, Z. (1993). Quelques resultats de stabilisation robuste: Applications a la commande, Ph.D. Thesis, Ecole des Mines de Paris.

[24] JiANG, Z.-P., HiLl, D. J. and FradKov, A. L. (1996). A passification approach to adaptive non-linear stabilization, Systems \& Control Letters, 28, 73-84.

[25] KaILATH, T. (1980). Linear Systems (Prentice-Hall).

[26] Kim, K., Ortega, R., Charara, A. and Vilain, J. P. (To appear). Theoretical and Experimental Comparison of Two Nonlinear Controllers for Current-fed Induction Motors, IEEE Trans. Cont. Syst. Tech.

[27] Krstić, M., Kanellakopoulos, I. and KoKotović, P. V. (1995). Nonlinear and Adaptive Control Design, ISBN 0-471-12732-9 (John Wiley \& Sons).

[28] KolESNIKOV, A. (1987) Analytical construction of nonlinear agregated regulators from a given set of invariant manifolds, Izvestia Vuishix Uchebnix Zavedenii: Electromexanika, 3, 100-108.

[29] Kокотоvic, P. V. (1992). The joy of feedback, IEEE Cont. Syst. Mag., 12, 7-17.

[30] Krause, P. C. (1986). Analysis of Electric Machinery (McGraw-Hill).

[31] KRENER, A. J. (1992). Optimal Model Matching Controllers for Linear and Nonlinear Systems, Proc. IFAC NOLCOS Conf., Bordeaux, France.

[32] Krstic, M., Kanellakopoulos I. and KокоTOVic, P. (1994). Passivity and parametric robustness of a new class of adaptive systems, Automatica, 30, 1703-1716.

[33] KuCERA, V. (1991). Analysis and design of discrete linear control systems (Prentice Hall, NY).

[34] LanCaster P. and Tismenetsky, M. The Theory of Marrices, Academic Press, 1985.

[35] LANARI, L. and WEN, J. T. (1992). Adaptive PD controller for robot manipulators, Syst. \& Contr. Letters, 19, 119-129.

[36] LeONHARD, W. (1996). Control of Electrical Drives, 2nd edn. (Springer-Verlag).

[37] LIPO, T. (1991). Synchronous Reluctance Machines-A Viable Alternative for AC Drives, Electric Machines and Power Systems, 19, 659-672.

[38] Liu, X., VergheSE, G., LANG, J. and ÖNDER, M. (1989). Generalizing the Blondel-Park Transformation of Electrical Machines: Necessary and Sufficient Conditions, IEEE Trans. Cir. Syst., 36, 1085-1067.

[39] LORENZ, R. D., LIPO, T. A. and NovotnY, D. W. (1994). Motion Control with Induction Motors, Proc. of the IEEE, (Special Issue on Power Electronics and Motion Control), 82, 1215-1240.

[40] Lozano, R., Brogliato, B. and Landau, I. (1992). Passivity and global stabilization of cascaded nonlinear systems, IEEE Trans. Aut. Control, 37, 1386-1388.

[41] Marino, R., Pereseda, S. and Valigi, P. (1993). Adaptive Input-Output Linearizing Control of Induction Motors, IEEE Trans. Aut. Control, 38, 208-221.

[42] Marino, R. and TomeI, P. (1991). Dynamic output feedback linearization and global stabilization, Syst. \& Control Letters, 17, 15-21.

[43] Marino, R. and TOMEI, P. (1995). Nonlinear Control Design, Geometric, Adaptive and Robust, ISBN 0-13-342635-1 (Prentice Hall).

[44] MeISEL, J. (1996). Principles of Electromechanical-Energy Conversion (McGraw-Hill). Miller, T. J. E. (reprint). Brushless Permanent-Magnet and Reluctance Motor Drives (Oxford, Clarendon Press).

[45] NAM, K. and ARAPOSTATHIS, A. (1988). A MRAC scheme for pure feedback systems, IEEE Trans. Aut. Control, 33, 803-811.

[46] NeĬMARK, J. I. and FufAEV, N. A. (1972). Dynamics of nonholonomic Systems, Vol. 33 of Translations of Mathematical Monographs, American Mathematical Society.

[47] Nicklasson, P. J. (1996). Passivity-Based Control of Electric Machines, Ph.D. Thesis, ISBN 82-7119-905-6, NTH, Norway.

[48] NiJMeIJeR, H. and VAN DER SCHAFT, A. (1990). Nonlinear Dynamical Control Systems (Springer Verlag). 
[49] Ogasawara, S., AKagi, H. and Nabae, A. (1988). The Generalized theory of Indirect Vector Control for AC Machines, IEEE Trans. Indust. Appl., 24, 470-478.

[50] ORTEGA, R. (1991). Passivity properties for stabilization of cascaded nonlinear systems, Automatica, 27, 423-424.

[51] ORTEGA, R. and SPONG, M. (1989). Adaptive motion control of rigid robots: A tutorial, Automatica, 25, 877-888.

[52] Ortega, R., Nicklasson, P. J. and Espinosa-Pérez, G. (1996). On Speed Control of Induction Motors, Automatica, 32, 455-460.

[53] Panteley, E. and Ortega, R. (To appear). Output feedback global tracking of interconnected Lagrangian systems, Automatica.

[54] PozharitskiI, G. K. (1962). On Asymptotic Stability of Equilibria and Stationary Motions of Mechanical Systems and Partial Dissipation, Prikl. Mat. $i$ Mekh., 1961, 25, 657-667. Engl. transl. in J. Appl. Math. Mech., 25.

[55] SONTAG, E. (1995). State-space and I/O stability for nonlinear systems, Feedback Control, Nonlinear Systems, and Complexity, Lecture Notes in Control and Information Sciences, 202, (Berlin, Springer-Verlag). (Eds) B. A. FranCIS and A. R. TANNENBAuM.

[56] RodrigueZ, A. and OrTEGA, R. (1990). Adaptive control of nonfeedback linearizable systems, Proc. 11th World IFAC Cong., Aug. 13-17, Tallinn, USSR, 4, 121-124.

[57] SEELY, S. (1962). Electromechanical Energy Conversion (McGraw-Hill).

[58] Seron, M. M., Hill, D. J. and Fradkov, A. L. (1995). Nonlinear adaptive control of feedback passive systems, Automatica, 31, 1053-1060.

[59] Sira-Ramirez, H., Ortega, R., Perez, R. and Garcia, M. (1995). Passivity-based controllers for the stabilization of DC-to-DC power converters, Proc. 34th IEEE CDC, New Orleans, LA, 3471-3476. (Also to appear in Automatica).

[60] SPONG, M. and VidyASAgar, M. (1989). Robot dynamics and control (John Wiley and Sons).

[61] TAYLOR, D. G. (1994). Nonlinear Control of Electric Machines: An Overview, IEEE Control Systems Magazine, 14, 41-51.

[62] TAKEgAKI, M. and ARIMOTO, S. (1981). A new feedback method for dynamic control of manipulators, ASME J. Dyn. Syst. Meas. Cont., 102, 119-125.

[63] TOMEI, P. (1991). A simple PD controller for robots with elastic joints, IEEE Trans. Aut. Cont., 36, 1208-1213.

[64] VAN DER SCHAFT, A. (1996). $\mathscr{L}_{2}$-Gain and Passivity Techniques in Nonlinear Control, Vol. 218 (Springer-Verlag, LNCINS).

[65] VAS, P. (1990). Vector Control of AC Machines (Oxford, Clarendon Press).

[66] Verghese, G. C., LANG, J. H. and CASEY, L. F. (1986). Analysis of Instability in Electrical Machines, IEEE Trans. Indust. Appl., 22, 853-864.

[67] White, D. C. and Woodson, H. H. (1959). Electromechanical Energy Conversion (New York, Wiley).

[68] WiLLEMS, J. L. (1972). A system theory approach to unified electrical machine analysis, Int. J. Contr., 15, 401-418.

[69] DE Wit, P., ORTEGA, R. and MAREELs, I. (1995). Indirect Field Oriented Control of Induction Motors is Robustly Globally Stable, Proc. 34th IEEE CDC, New Orleans, LA, 2139-2144. (Also to appear in Automatica).

[70] Youla, D. C. and Bongiorno, J. J. (1980). A Floquet theory of the general rotating machine, IEEE Trans. Circuits Syst., CAS-27, 15-19.

[71] ZRIBI, M. and ChIasson, J. (1991). Position Control of a PM Stepper Motor by Exact Linearization, IEEE Trans. Aut. Cont., 36, 620-625. 